\title{
Adaptive Quasi-PID Control Method for Switching Power Amplifiers
}

\author{
Xiaoming Sun \\ Department of Electrical Engineering, Chongqing Water Resources and Electric Engineering College, Chongqing, China \\ Email:xmusn.whu@163.com
}

How to cite this paper: Sun, X.M. (2017) Adaptive Quasi-PID Control Method for Switching Power Amplifiers. Journal of Power and Energy Engineering, 5, 19-44. https://doi.org/10.4236/jpee.2017.52003

Received: January 4, 2017

Accepted: February 6, 2017

Published: February 9, 2017

Copyright (C) 2017 by author and Scientific Research Publishing Inc. This work is licensed under the Creative Commons Attribution International License (CC BY 4.0).

http://creativecommons.org/licenses/by/4.0/

\begin{abstract}
Quasi-PID control method that is able to effectively inhibit the inherent tracking error of PI control method is proposed on the basis of a rounded theoretical analysis of a model of switching power amplifiers (SPAs). To avoid the harmful impacts of the circuit parameter variations and the random disturbances on quasi-PID control method, a single neuron is introduced to endow it with self-adaptability. Quasi-PID control method and the single neuron combine with each other perfectly, and their formation is named as single-neuron adaptive quasi-PID control method. Simulation and experimental results show that single-neuron adaptive quasi-PID control method can accurately track both the predictable and the unpredictable waveforms. Quantitative analysis demonstrates that the accuracy of single-neuron adaptive quasiPID control method is comparable to that of linear power amplifiers (LPAs) and so can fulfill the requirements of some high-accuracy applications, such as protective relay test. Such accuracy is very difficult to be achieved by many modern control methods for converter controls. Compared with other modern control methods, the programming realization of single-neuron adaptive quasi-PID control method is more suitable for real-time applications and realization on low-end microprocessors for its simple structure and lower computational complexity.
\end{abstract}

\section{Keywords}

Switching Power Amplifier, Quasi-PID Control Method, Single Neuron, Adaptive Control, Protective Relay Test

\section{Introduction}

Generating and amplifying waveforms with medium power (i.e., from 1 or $2 \mathrm{~W}$ to 1 or $2 \mathrm{~kW}$ ) have many important applications in various industrial fields, such as protective relay test, and audio process. The task of generating and amplifying 
a waveform is tracking the command signal of the waveform in current form and voltage form. An amplifier designed for current tracking is called as a current amplifier, and that designed for voltage tracking is called as a voltage amplifier.

Apparently, it is easy to generate and amplify a wave-form accurately with low power (i.e., less than 1 or $2 \mathrm{~W}$ ), but, with medium power, the accuracy is difficult to control. So, linear power amplifiers (LPAs) [1] that consist of high-power transistors are widely used to retain the linear relationships between the command signals and the output waveforms to acquire a high tracking accuracy. However, with the development of power electronics technology, switching power amplifiers (SPAs) based on converters (including rectifiers and inverters) are also used in a good many waveform generation and amplification occasions, such as active power filters (APFs), and low-fidelity audio amplifiers.

Compared with LPAs, SPAs have those advantages: 1) SPAs do not need the digital-to-analog converters that are sometimes very expensive; 2) the nominal capacity of a switching device is usually much higher than that of a high-power transistor, and thus there is no need to parallel or cascade several devices to obtain a high output power in SPAs, implying a high performance-price ratio of SPAs; 3) unlike LPAs, which need at least 3 stages to obtain a high amplifying gain, traditionally, SPAs need only 1 amplifying stage, meaning that the basic architecture of SPAs is much simpler; 4) the efficiency of SPAs is much higher than that of LPAs because the devices operate in a high-speed switching state; 5) it is easy to isolate the digital signals from the high-power output signals in SPAs by photoelectric couplers.

Although SPAs have the advantages above, the tracking accuracy of SPAs is harder to control than LPAs. To improve the tracking accuracy of SPAs, the authors tested some modern control methods for converter controls. Repetitive control method [2] [3] [4], which is based on the internal model principle and is a high-performance feed forward control strategy, can effectively track the periodic signals and eliminate the periodic disturbances or distortions. However, when the command signal is nonperiodic or unpredictable, the dynamic response becomes slow, and the tracking accuracy degrades significantly. Deadbeat control method [5] [6], which is a superior predictive control strategy, has excellent dynamic response and good transient tracking accuracy. However, the actual tracking accuracy depends greatly on its predictive model, the choice of which is empirical and subjective, and thus it is difficult to ensure the optimality of the predictive model. Moreover, the predictive model is sensitive to the uncertainties of the control object, e.g. the parameter variations of the load, which sometimes influence the tracking accuracy. Sliding mode control method [7] [8] [9] shows a good robustness against system parameter variations once the operating point enters the predefined sliding surface. However, it is difficult to design an optimal sliding surface that can adapt to all types of situations. In addition, it is based on an ideal assumption that the sliding velocity of the operating point is infinitely fast, which is unattainable in practical implementations due to 
the switching frequency limitations of the devices and other factors. These problems always induce oscillations in the output waveforms. Moreover, without complex improvements, it may suffer from great switching frequency variations. In short, these control methods are more suitable for generating and amplifying deterministic waveforms to deterministic loads (e.g. in frequency converters), or tracking various frequency components with relatively low accuracy (e.g. in APFs). Their applications in high-accuracy and variable-load fields are usually limited.

In the process of testing the control methods above to find out the most favorable one for generating and amplifying waveforms with unpredictable characters to variable loads with high-accuracy, the authors discovered an interesting control method, which inherits certain characteristics of both PID control method and deadbeat control method. Because it is more similar to PID control method, it is called quasi-PID control method. Further study shows that quasiPID control method can be integrated with a single neuron perfectly, so the self-adaptability to variable loads and self-adjustment to random errors can be achieved conveniently. It is called single-neuron adaptive quasi-PID control method, and this paper focuses on discussing its derivation details and its application in SPAs for protective relay test.

\section{Modeling of an SPA}

The SPA discussed in this paper is based on a single-phase full-bridge topology and an independent DC source (shown in Figure 1), which can be combined as independent blocks to obtain multiple-channel outputs.

\subsection{Open-Loop Model}

In Figure 1, Q1-Q4 are insulated-gate bipolar transistors (IGBTs), $D 1-D 4$ are fast-recovery free-wheeling diodes, $L$ and $C$ are inductor and capacitor of $L C$ output filter, $R$ is a load resistor, $\bar{V}_{d c}$ (a constant) is average voltage of DC

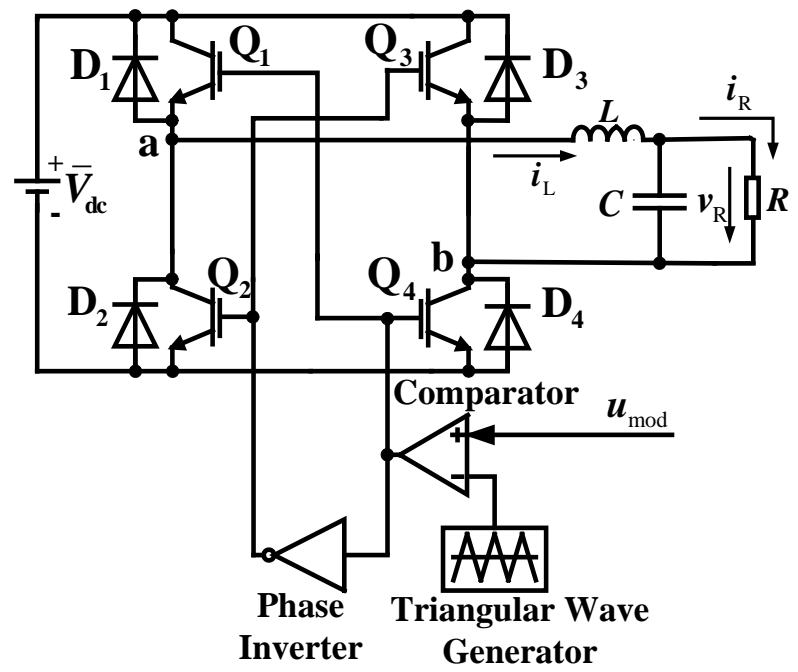

Figure 1. Circuit topology of an SPA. 
source, $i_{L}$ is inductor current, $i_{R}$ is load current, $v_{R}=i_{R} R$ is load voltage and $u_{\text {mod }}$ is modulation signal. The snubber circuits of SPA are omitted for simplicity, the design of which can be found in [10].

Figure 2 illustrates the principle of generating the bipolar pulse width modulation (PWM) signals, where $V_{c}$ is the amplitude of the isosceles-triangle carrier, $T_{s}$ is the carrier period and also the switching period and the sampling period (the sampling frequency $f_{s}=1 / T_{s}$ ). According to the equivalent-area principle [11] [12], the area of the curved-edge trapezoidal pulse ABCDE should be equal to the net area of the PWM pulses, i.e. $S_{B}=S_{B 1}-S_{B 2}-S_{B 3}$. Because $T_{S}$ is very small, the area of $\mathrm{ABCDE}$ is close to the area of the rectangle $A^{\prime} C^{\prime} D E$ (the shadowed area $\left.S_{A}\right) . S_{A}$ and $S_{B}$ can be written as

$$
\begin{aligned}
& S_{A}=u_{\text {modc }} T_{s} \\
& S_{B}=S_{B 1}-S_{B 2}-S_{B 3}=\bar{V}_{d c} t_{\text {on }}-\bar{V}_{d c} t_{\text {off }}-\bar{V}_{d c} t_{\text {off }}
\end{aligned}
$$

where $u_{\text {modc }}$ is the ordinate of the intersection point $B, t_{\text {on }} \in\left[0, T_{s}\right]$ and $t_{\text {off }} \in\left[0, T_{s} / 2\right]$ are the turn-on time and turn-off time of $Q 1$ and $Q 4$. From $S_{A}=S_{B}$, the following relationship is obtained:

$$
u_{\text {modc }}=\left(t_{\text {on }}-T_{s} / 2\right)\left(2 \bar{V}_{d c} / T_{s}\right)
$$

where the relationship $t_{\text {on }}=T_{s}-2 t_{\text {off }}$ is considered. The prerequisite for accurate tracking is that the average output voltage $\bar{v}_{a b}$ between points $a$ and $b$ (seen in Figure 1) should be equal to $u_{\text {modc }}$, thus

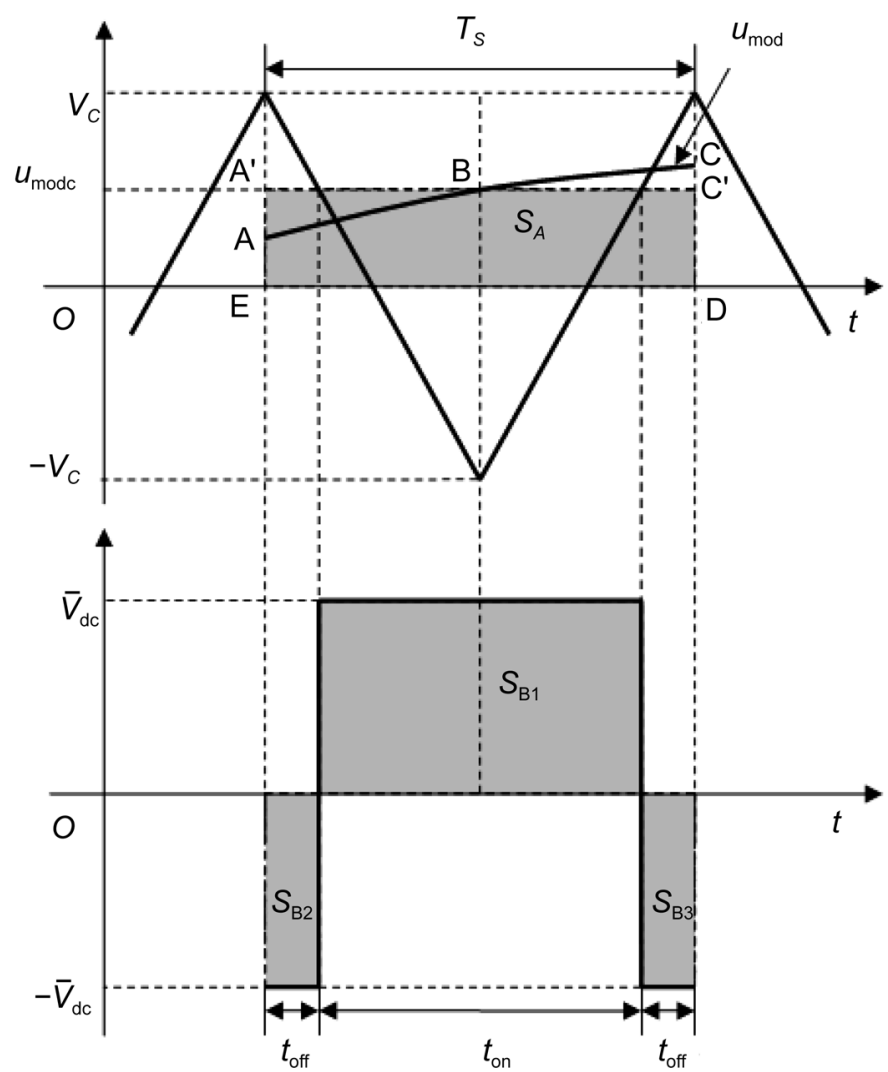

Figure 2. Principle of generating the bipolar PWM signals. 


$$
\bar{v}_{a b}=u_{\text {modc }}=t_{\text {bon }} K_{t v}, t_{\text {bon }} \in\left[-T_{s} / 2, T_{s} / 2\right]
$$

where $t_{\mathrm{bon}}=t_{\mathrm{on}}-T_{s} / 2(\mathrm{~s})$ and $K_{t v}=2 \bar{V}_{d c} / T_{s}(\mathrm{~V} / \mathrm{s}) . t_{\mathrm{bon}}$ is called biased turn-on time (with an offset $-T_{s} / 2$ ), and $K_{t v}$ is called time-to-voltage transfer coefficient. It is easy to know that: 1$)$ if $t_{\text {on }}=0\left(t_{\text {bon }}=-T_{s} / 2\right)$, then $\left.\bar{V}_{a b}=-\bar{V}_{d c} ; 2\right)$ if $t_{\text {on }}=T_{s} / 2\left(t_{\text {bon }}=0\right)$, then $\left.\bar{v}_{a b}=0 ; 3\right)$ if $t_{\text {on }}=T_{s}\left(t_{\text {bon }}=T_{s} / 2\right)$, then $\bar{v}_{a b}=\bar{V}_{d c}$. So, Equation (4) excellently describes the whole process of turn-on and turn-off, and is the correct open-loop model of SPA.

\subsection{Closed-Loop Model}

To realize the closed-loop control, the output of SPA should be fed back to affect $u_{\text {mod }}$, and there are 2 ways to do so: 1$)$ let $\left.u_{\text {mod }}=K_{i e} e_{i}=K_{i e}\left(i_{R}^{*}-i_{R}\right) ; 2\right)$ let $u_{\text {mod }}=K_{v e} e_{v}=K_{v e}\left(v_{R}^{*}-v_{R}\right) \cdot i_{R}^{*}$ is the current command signal, $v_{R}^{*}$ is the voltage command signal, $e_{i}$ is the output current deviation, $e_{v}$ is the output voltage deviation, $K_{i e}$ is the current error amplification coefficient and $K_{v e}$ is the voltage error amplification coefficient. Because $u_{\bmod }$ can be assigned with either $K_{i e} e_{i}$ or $K_{v e} e_{v}$, the SPA in Figure 1 can realize either current tracking or voltage tracking, though it is supplied by a voltage source. Given that most of the references are dedicated to discussing voltage tracking [13] [14], and that the control method of current tracking can be transplanted to voltage tracking with slight modifications as discussed, this paper concentrates on discussing current tracking only.

\subsection{Continuous Model in Frequency Domain}

$t_{\text {bon }}$ in Equation (4) is the output of the controller and the output of SPA needs at least 1sampling period $T_{s}$ to be fed back to the controller, so the continuous model of SPA in frequency domain can be constructed as shown in Figure 3. The 3 blocks enclosed by the dash-dotted frame are the general model of SPA, which considers SPA, LC output filter and load resistor together:

$$
G(s)=\frac{I_{R}(s)}{T_{\text {bon }}(s)}=\frac{K_{t v} e^{-T_{s} s}}{R L C s^{2}+s L+R}
$$

$I_{R}^{*}(s), I_{R}(s)$ and $T_{\text {bon }}(s)$ are the Laplace transforms of $i_{R}^{*}, i_{R}$ and $t_{\text {bon }}$, $C(s)$ is the transfer function of the controller, and $F$ is the proportional coefficient of feedback channel.

Although it is easy to write out the closed-loop transfer function according to Figure 3, it is difficult to design the controller due to the pure-delay term $e^{-T_{s} s}$ which leads the system to be a non-minimum phase system [15]. Further, although the system can be turned into a minimum phase system by expanding $e^{-T_{s} s}$ into a power series and taking a finite number of the fore terms, this would suffer a great loss of the system bandwidth. Therefore, it is wise to design the controller from another angle, i.e., in time domain.

\subsection{Discrete Model in Time Domain}

For digital simulation in time domain, $G(s)$ must be discretized in time domain. 


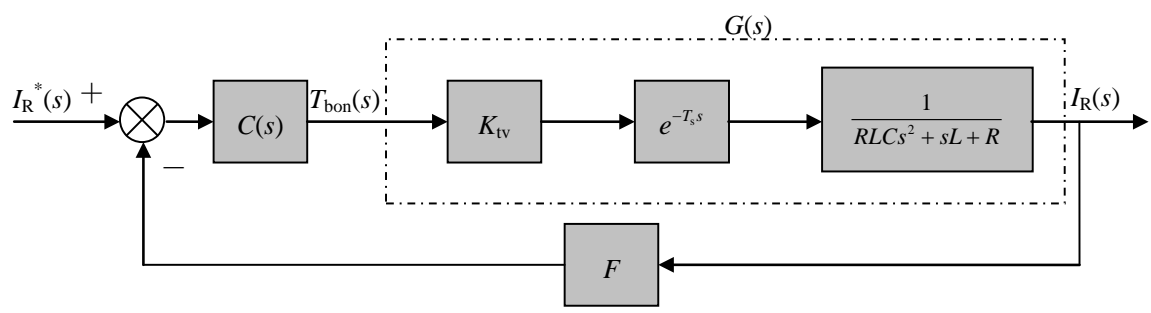

Figure 3. Continuous model of SPA in frequency domain.

The first step is to transform $G(s)$ in $s$ domain to $G(z)$ in $z$ domain by virtue of the relationship between Laplace transform and $z$ transform:

$$
G(z)=Z\left[\frac{1-e^{-T_{s} s}}{s} G(s)\right]=Z\left[\frac{1-e^{-T_{s} s}}{s} \frac{K_{t v} e^{-T_{s} s}}{R L C s^{2}+s L+R}\right]
$$

where $Z[\cdot]$ denotes performing $z$ transform on the expressions in the square brackets. To maintain the invariability of the system step response after $z$ transform, a zero-order holder, i.e., $\left(1-e^{-T_{s} s}\right) / s$, is introduced to Equation (6). $G(z)$ is very complex if expressed with parameter symbols, so, instead, it is expressed in numerical type with detailed values of the parameters substituted into the expression and calculated (the values of the parameters are listed in Appendix A):

$$
G(z)=\frac{I_{R}(z)}{T_{\text {bon }}(z)}=\frac{2.479 \times 10^{4} z^{-2}+1.845 \times 10^{4} z^{-3}}{1-1.315 z^{-1}+0.412 z^{-2}} .
$$

The second step is to perform inverse $z$ transform on $G(z)$ to get the difference equation:

$$
\begin{aligned}
i_{R}(k)= & 1.315 i_{R}(k-1)-0.412 i_{R}(k-2)+2.479 \times 10^{4} t_{\text {bon }}(k-2) \\
& +1.845 \times 10^{4} t_{\text {bon }}(k-3)
\end{aligned}
$$

where $k$ is the integer index of the discrete time series, $k=0,1,2,3, \cdots$. It is assumed: $i_{R}(-1)=i_{R}(-2)=0$ and $t_{\mathrm{bon}}(-1)=t_{\mathrm{bon}}(-2)=t_{\mathrm{bon}}(-3)=0$.

\section{Quasi-PID Control Method}

The Kirchhoff voltage and current equations of the SPA in Figure 1 are as follows:

$$
\begin{aligned}
& (2 p-1) \bar{V}_{d c}=L \frac{\mathrm{d} i_{L}}{\mathrm{~d} t}+i_{L} r+v_{R} \\
& i_{L}=C \frac{\mathrm{d} v_{R}}{\mathrm{~d} t}+i_{R}=R C \frac{\mathrm{d} i_{R}}{\mathrm{~d} t}+i_{R}
\end{aligned}
$$

where $r=r_{Q}+r_{L} \quad\left(r_{Q}\right.$ is the equivalent switching resistance of IGBT, $r_{L}$ is the winding resistance of $L$ ) and $p$ is a unipolar two-valued-logic switching function:

$$
p=\left\{\begin{array}{l}
1\left(\begin{array}{l}
Q_{1} \text { and } Q_{4} \text { ON or } D_{1} \text { and } D_{4} \text { ON } \\
Q_{2} \text { and } Q_{3} \text { OFF and } D_{2} \text { and } D_{3} \text { OFF }
\end{array}\right) \\
\left.0 \begin{array}{l}
Q_{2} \text { and } Q_{3} \text { ON or } D_{2} \text { and } D_{3} \text { ON } \\
Q_{1} \text { and } Q_{4} \text { OFF and } D_{1} \text { and } D_{4} \text { OFF }
\end{array}\right)
\end{array}\right.
$$

when the symmetric regular sampling method is adopted in the modulating 
process as shown in Figure 2, it is easy to write out the duty cycle

$$
D=\frac{t_{\mathrm{on}}}{T_{s}}=\frac{u_{\mathrm{modc}}+V_{c}}{2 V_{c}} \approx \frac{K_{i e} e_{i}}{2 V_{c}}+\frac{1}{2} .
$$

The duration time for $p=1$ is $t_{\text {on }}$ and that for $p=0$ is $t_{\text {off }}$.

Given that $T_{s}$ is very small, the integration of current differential $d i_{L}$ within a $T_{s}$ is equal to the summation of small current variations, which is approximate to inductor current variation $\Delta i_{L}$. Thus, by integrating both sides of Equation (9) over a $T_{s}$, an expression is obtained:

$$
\begin{aligned}
\Delta i_{L} & \approx \int_{0}^{T_{s}} \mathrm{~d} i_{L}=\frac{1}{L} \int_{0}^{T_{s}}\left[(2 p-1) \bar{V}_{d c}-\left(i_{L} r+v_{R}\right)\right] \mathrm{d} t \\
& =\frac{1}{L}\left\{2\left[-\bar{V}_{d c}-\left(i_{L} r+v_{R}\right)\right] t_{\text {off }}+\left[\bar{V}_{d c}-\left(i_{L} r+v_{R}\right)\right] t_{\text {on }}\right\} \\
& =\frac{T_{s}}{L}\left[(2 D-1) \bar{V}_{d c}-\left(i_{L} r+i_{R} R\right)\right]=\frac{T_{s}}{L}\left[\frac{K_{i e} e_{i} \bar{V}_{d c}}{V_{c}}-\left(i_{L} r+i_{R} R\right)\right]
\end{aligned}
$$

when $i_{L}$ is increasing, i.e., $\Delta i_{L} \geq 0$, from Equation (13)

$$
e_{i} \geq \frac{V_{c}\left(i_{L} r+i_{R} R\right)}{K_{i e} \bar{V}_{d c}}=e_{i}^{*} \neq 0
$$

where $e_{i}^{*}$ is defined as the inherent tracking error. Likewise, when $i_{L}$ is decreasing, i.e., $\Delta i_{L} \leq 0$, Equation (14) becomes $e_{i} \leq e_{i}^{*} \neq 0$. Here, $e_{i}^{*} \neq 0$ indicates that $e_{i}$ is fluctuating around a nonzero value, that is to say, the non-staticerror tracking cannot be realized.

To counteract the nonzero $e_{i}^{*}$, the authors creatively construct a modified current command signal:

$$
\hat{i}_{R}^{*}=i_{R}^{*}+e_{i}^{*}=i_{R}^{*}+\frac{V_{c}\left(i_{L} r+i_{R} R\right)}{K_{i e} \bar{V}_{d c}} .
$$

By replacing the $i_{R}^{*}$ in $e_{i}\left(e_{i}=i_{R}^{*}-i_{R}\right)$ in Equations (12) and (13) with $\hat{i}_{R}^{*}$, the modified duty cycle $\hat{D}$ and the modified inductor current variation $\Delta \hat{i}_{L}$ are written as

$$
\begin{aligned}
& \hat{D}=\frac{K_{i e}\left(\hat{i}_{R}^{*}-i_{R}\right)}{2 V_{c}}+\frac{1}{2}=\frac{K_{i e} e_{i}}{2 V_{c}}+\frac{i_{L} r+i_{R} R}{2 \bar{V}_{d c}}+\frac{1}{2} \\
& \Delta \hat{i_{L}} \approx \frac{T_{s}}{L}\left[\frac{K_{i e}\left(\hat{i}_{R}^{*}-i_{R}\right) \bar{V}_{d c}}{V_{c}}-\left(i_{L} r+i_{R} R\right)\right]=\frac{e_{i} T_{s} K_{i e} \bar{V}_{d c}}{L V_{c}} .
\end{aligned}
$$

According to Equation (17), whether $\Delta \hat{i}_{L}$ is increasing or decreasing, $e_{i}$ is fluctuating around 0 now, and thus the inherent tracking error is eliminated.

If the coefficient of $e_{i}$ in Equation (17) is intentionally forced to be equal to 1 , then

$$
K_{i e}=\frac{L V_{c}}{T_{s} \bar{V}_{d c}}=\frac{L V_{c} f_{s}}{\bar{V}_{d c}}
$$

and this leads to a concise form of Equation (17):

$$
\Delta \hat{i}_{L} \approx e_{i} .
$$


In practice, the duty cycle is the final control quantity of SPAs, and it needs to be discretized for digital control, which entails the discretization of Equation (16):

$$
\hat{D}(k)=\frac{K_{i e} e_{i}(k)}{2 V_{c}}+\frac{i_{L}(k) r+i_{R}(k) R}{2 \bar{V}_{d c}}+\frac{1}{2}
$$

and the incremental type, i.e., $\Delta \hat{D}(k)=\hat{D}(k)-\hat{D}(k-1)$ is

$$
\begin{aligned}
\Delta \hat{D}(k)= & \frac{K_{i e}}{2 V_{c}}\left[e_{i}(k)-e_{i}(k-1)\right]+\frac{r}{2 \bar{V}_{d c}}\left[i_{L}(k)-i_{L}(k-1)\right] \\
& +\frac{R}{2 \bar{V}_{d c}}\left[i_{R}(k)-i_{R}(k-1)\right] .
\end{aligned}
$$

Similarly, Equation (10) is discretized as

$$
i_{L}(k)=\frac{R C}{T_{s}}\left[i_{R}(k)-i_{R}(k-1)\right]+i_{R}(k)
$$

where the first-order backward difference is adopted to approximate the firstorder differential. The incremental type of Equation (22), i.e.,

$$
\Delta i_{L}(k)=i_{L}(k)-i_{L}(k-1)
$$

is

$$
\Delta i_{L}(k)=\frac{R C}{T_{s}}\left[i_{R}(k)-2 i_{R}(k-1)+i_{R}(k-2)\right]+i_{R}(k)-i_{R}(k-1) .
$$

And the discretized type of Equation (19) is

$$
\Delta \hat{i}_{L}(k)=i_{L}(k)-i_{L}(k-1) \approx e_{i}(k) .
$$

Substitute Equation (24) into Equation (23), Equation (23) can be rearranged as

$$
i_{R}(k)-i_{R}(k-1)=e_{i}(k)-\frac{R C}{T_{s}}\left[i_{R}(k)-2 i_{R}(k-1)+i_{R}(k-2)\right] .
$$

Then substitute Equations (24) and (25) into Equation (21), Equation (21) becomes

$$
\begin{aligned}
\Delta \hat{D}(k)= & \frac{K_{i e}}{2 V_{c}}\left[e_{i}(k)-e_{i}(k-1)\right]+\frac{r+R}{2 \bar{V}_{d c}} e_{i}(k) \\
& -\frac{R^{2} C}{2 \bar{V}_{d c} T_{s}}\left[i_{R}(k)-2 i_{R}(k-1)+i_{R}(k-2)\right] .
\end{aligned}
$$

A widely used type of PID control method [15] is

$$
u(k)=K_{P} e(k)+K_{I} \sum_{j=0}^{k} e(j) T_{s}+\frac{K_{D}}{T_{s}}[e(k)-e(k-1)] .
$$

where $u(k)$ is the control quantity, $e(k)$ is the error between the real output and the expected output (command signal), $K_{P} K_{I}$ and $K_{D}$ are $P, I$ and $D$ parameters. The incremental type of Equation (27), i.e., $\Delta u(k)=u(k)-u(k-1)$ is

$$
\begin{aligned}
\Delta u(k)= & K_{P}[e(k)-e(k-1)]+K_{I} T_{s} e(k) \\
& +\frac{K_{D}}{T_{s}}[e(k)-2 e(k-1)+e(k-2)] .
\end{aligned}
$$


A term-to-term comparison between Equations (26) and (28) discloses that the first 2 terms are in accordance with each other, and the third term of Equation (26) is composed of $i_{R}(k)$ while that of Equation (28) is composed of $e(k)$. Therefore, Equation (28) is not a real PID controller, yet it does have a structure similar to that of a PID controller. Due to this, Equation (28) is called as quasi-PID control method. From the comparison, it is easy to write out the quasi-PID parameters:

$$
K_{P}=\frac{K_{i e}}{2 V_{c}}=\frac{L f_{s}}{2 \bar{V}_{d c}}, K_{I}=\frac{(r+R) f_{s}}{2 \bar{V}_{d c}}, \hat{K}_{D}=\frac{-R^{2} C}{2 \bar{V}_{d c}}
$$

where the quasi- $D$ parameter is denoted as $\hat{K}_{D}$ to be distinguished from $K_{D}$. Accordingly, Equation (26) is simplified as

$$
\begin{aligned}
\Delta \hat{D}(k)= & K_{P}\left[e_{i}(k)-e_{i}(k-1)\right]+K_{I} T_{s} e_{i}(k) \\
& +\frac{\hat{K}_{D}}{T_{s}}\left[i_{R}(k)-2 i_{R}(k-1)+i_{R}(k-2)\right] .
\end{aligned}
$$

Considering that the control quantity in Equation (8) is $t_{\text {bon }}(k), \Delta \hat{D}(k)$ must be converted to $\Delta t_{\text {bon }}(k)$ :

$$
\begin{aligned}
\Delta t_{\text {bon }}(k)= & t_{\text {bon }}(k)-t_{\text {bon }}(k-1)=\Delta t_{\text {on }}(k)=T_{s} \Delta \hat{D}(k) \\
= & K_{P} T_{s}\left[e_{i}(k)-e_{i}(k-1)\right]+K_{I} T_{s}^{2} e_{i}(k) \\
& +\hat{K}_{D}\left[i_{R}(k)-2 i_{R}(k-1)+i_{R}(k-2)\right] .
\end{aligned}
$$

\section{Single-Neuron Adaptive Quasi-PID Control Method}

Equation (29) shows that all 3 quasi-PID parameters are related to the circuit parameters $L, f_{s}, \bar{V}_{d c}, r, R$ and $C$. These "known" parameters actually vary with loads, operating conditions and disturbances. For example: (i) the fluctuation of the output power would lead to the fluctuation of $\bar{V}_{d c}$, so the presumption that $\bar{V}_{d c}$ is a constant should be discounted; (ii) the resistance of $R$ is always drifting with the load temperature; (iii) the nonlinear variations of $r_{Q}$ and $r_{L}$ may make $r$ ripple nonlinearly. All these issues would influence the accuracy of the quasi-PID parameters and further degrade the tracking accuracy. In addition, the dead-time embedded in turn-on time and the side effect of snubber circuits may introduce extra errors. The authors found that quasi-PID control method can be integrated with a single neuron perfectly, and so the adaptive online adjustment of the quasi-PID parameters can be realized conveniently, making the dynamic compensations for the aforementioned detrimental influences and extra errors feasible.

\subsection{Adaptive Control Structure}

The structure of single-neuron adaptive quasi-PID control method is presented in Figure 4, where $x_{1}(k), x_{2}(k)$ and $x_{3}(k)$ are the 3 inputs of the single neuron: 


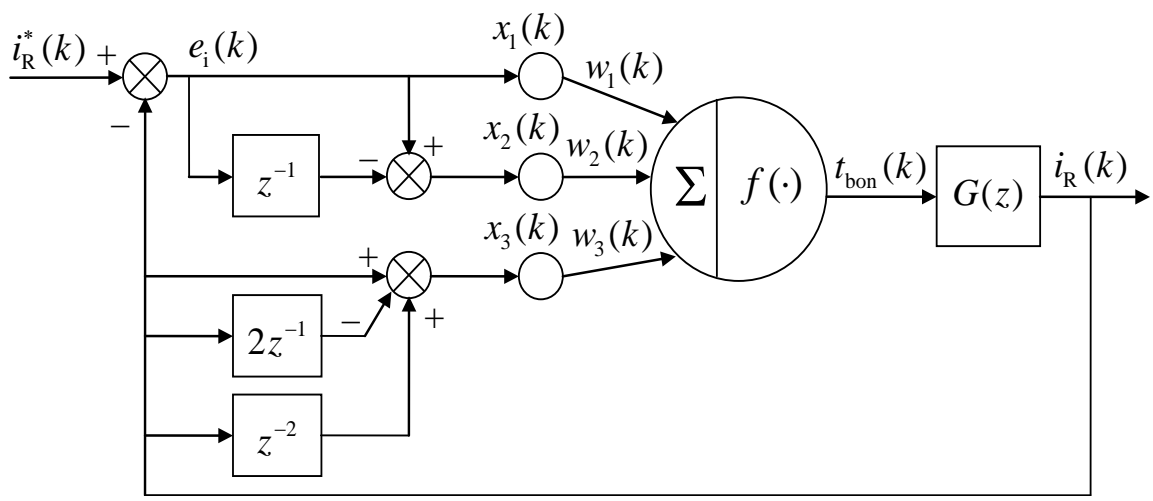

Figure 4. Structure of single-neuron adaptive quasi-PID control method.

$$
\left\{\begin{array}{l}
x_{1}(k)=e_{i}(k)-e_{i}(k-1) \\
x_{2}(k)=e_{i}(k)=i_{R}^{*}(k)-i_{R}(k) \\
x_{3}(k)=i_{R}(k)-2 i_{R}(k-1)+i_{R}(k-2)
\end{array}\right.
$$

And $w_{1}(k), w_{2}(k)$ and $w_{3}(k)$ are the 3 connection weights:

$$
w_{1}(k)=K_{P} T_{s}, w_{2}(k)=K_{I} T_{s}^{2}, w_{3}(k)=\hat{K}_{D} \text {. }
$$

The single neuron sums the 3 weighted inputs up by its adder component " $\Sigma$ " to form a total input signal:

$$
S_{\text {in }}(k)=\sum_{j=1}^{3} w_{j}(k) x_{j}(k)=\Delta t_{\text {bon }}(k) .
$$

Substitute Equations (32) and (33) into Equation (34), it is seen that Equation (34) actually realizes the same calculation of Equation (31).

The 3 connection weights in Equation (34) should be normalized to maintain their relative magnitudes to promote the robustness of simulation and actual control. The normalization can be carried out by virtue of vector norms. There are 3 commonly used vector norms [16]: 1) 1-norm, the summation of the absolute values of the elements; 2) 2-norm, the square root of the quadratic sum of the elements; 3) $\infty$-norm, the maximum value of the absolute values of the elements. Comparisons show that 2-norm is of the greatest computational complexity, and simulations show that it does not give a better control effect than 1 -norm. Although $\infty$-norm is of the lowest computational complexity, it always makes one of the 3 normalized connection weights equal to 1 , causing the corresponding input to have the greatest impact on the control quantity and thus inducing oscillations on the output waveform during the first 1 or 2 power frequency periods. Therefore, 1-norm is the best choice, and the normalized type of Equation (34) based on 1-norm is

$$
S_{\text {in(norm) }}(k)=\sum_{j=1}^{3} \frac{w_{j}(k)}{\|w(k)\|_{1}} x_{j}(k)=\sum_{j=1}^{3} \frac{w_{j}(k)}{\sum_{n=1}^{3}\left|w_{n}(k)\right|} x_{j}(k)=\sum_{j=1}^{3} \bar{w}_{j} x_{j}(k)
$$

where $\boldsymbol{w}(k)=\left[w_{1}(k), w_{2}(k), w_{3}(k)\right]$ is the connection weight vector, $\|\boldsymbol{w}(k)\|_{1}$ is the 1 -norm of $\boldsymbol{w}(k)$, and $\bar{w}_{j}$ is defined to replace the coefficient of $x_{j}(k)$ 
for short.

The single neuron takes $S_{\text {in(norm) }}(k)$ through excitation function $f(\cdot)$ to generate the normalized control quantity:

$$
\Delta t_{\text {bon(norm) }}(k)=f\left[S_{\text {in(norm) }}(k)\right]= \begin{cases}5 & S_{\text {in(norm) }}(k) \in(5, \infty) \\ K_{s l} S_{\text {in(norm) }}(k) & S_{\text {in(norm) }}(k) \in[-5,5] \\ -5 & S_{\text {in(norm) }}(k) \in(-\infty,-5)\end{cases}
$$

where a linear proportional function with amplitude limitations is chosen as $f(\cdot)$, and $K_{s l}$ is the slope of the linear segment of $f(\cdot)$. The choice of this excitation function lies on 2 considerations: (i) limiting the amplitude of the control quantity is indispensable to prevent the control quantity from overreaching; (ii) $S_{\text {in (norm) }}(k)$ has already been the required control quantity, further processes with complex excitation functions (such as the sigmoid function or the radial basis function) may not only deprive its physical meanings but induce unnecessary computational complexities, so it is better to choose a simple function to slightly adjust its amplitude. It should also be noted that the amplitude limitations of $f(\cdot)$ are set as \pm 5 instead of \pm 1 ( \pm 1 are the amplitude limitations defined in normalization theory). The reasons are: 1) avoiding pure decimalfraction computations on fixed point microprocessor used in this paper, which may introduce large rounding errors to the calculated data; 2) slightly loosening the amplitude limitations to enhance the fault tolerance of the algorithm.

In Equation (8), the coefficients of $t_{\text {bon }}(k-2)$ and $t_{\text {bon }}(k-3)$ are far greater than those of $i_{R}(k-1)$ and $i_{R}(k-2)$ because the variables and their coefficients are all actual values. Seeing that Equations (35) and (36) are in normalized types, Equation (8) cannot be calculated with them if not normalized accordingly. So 2 steps are taken to normalize $t_{\text {bon }}(k)$ and $i_{R}(k):$ (i) according to Equations (4) and (36) $t_{\text {bon }} \in\left[-T_{s} / 2, T_{s} / 2\right], t_{\text {bon(norm) }}(k) \in[-5,5]$, when $T_{s}=1 \times 10^{-4} \mathrm{~s}, t_{\text {bon }}(k) \in\left[-5 \times 10^{-5} \mathrm{~s}, 5 \times 10^{-5} \mathrm{~s}\right]$, so $t_{\text {bon }}(k)=t_{\text {bon(norm) }}(k) \times 10^{-5}$; (ii) the peak-to-peak values of the output current in this paper are designed as $\pm 10 \mathrm{~A}$, so $i_{R}(k)$ is normalized by being divided by $10 \mathrm{~A}$, and the result is the per-unit value $i_{R(\text { norm })}(k)=i_{R}(k) / 10$. Based on the 2 steps above, Equation (8) is normalized as

$$
\begin{aligned}
i_{R(\text { norm })}(k)= & 13.15 i_{R(\text { norm })}(k-1)-4.12 i_{R(\text { norm })}(k-2) \\
& +0.2479 t_{\text {bon(norm) }}(k-2)+0.1845 t_{\text {bon(norm) }}(k-3) .
\end{aligned}
$$

\subsection{Adaptive Learning Algorithm}

The general learning rule [17] for connection weight adjustment is as follows:

$$
\Delta w=\eta g(\boldsymbol{w}, \boldsymbol{x}, d) \boldsymbol{x}-\lambda \boldsymbol{w}
$$

where $\boldsymbol{w}$ is the connection weight vector, $\Delta \boldsymbol{w}$ is the incremental vector of $\boldsymbol{w}$, $\eta>0$ is the learning rate, $\boldsymbol{x}$ is the input vector, $d$ (a scalar quantity) is the expected output and is called the teacher signal, function $g(\cdot)$ is the learning signal and $\lambda \geq 0$ is a real constant. 
Argument 1: If $g(\boldsymbol{w}, \boldsymbol{x}, d)=d-y=e \quad(e$ is the error between $d$ and the actual output $y, e$ and $y$ are scalar quantities) and $\lambda=0$, then $\Delta \boldsymbol{w}=\eta(d-y) \boldsymbol{x}$, which is perceptron learning rule based on least mean square standard. This learning rule includes $d$, so it is a supervised learning rule with teacher guidance, and theory [18] verifies that it is asymptotically stable. The expanded type is

$$
\Delta w_{j}(k)=\eta[d(k)-y(k)] x_{j}(k)=\eta_{j} e(k) x_{j}(k)
$$

where, in this paper, $d(k)=i_{R(\text { norm })}^{*}(k), y(k)=i_{R(\text { norm })}(k), e(k)=e_{i}(k)$ and $j=1,2,3$. Simulations show that this learning rule possesses outstanding stability but lacks "independence" or "self-learning enthusiasm". When illustrated on the output waveform, the phenomenon is that the steady-state errors of the output waveform are very small while the response speed is fairly slow.

Argument 2: If $g(\boldsymbol{w}, \boldsymbol{x}, d)=u \quad(u$ is the control quantity and a scalar quantity) and $\lambda=0$, then $\Delta \boldsymbol{w}=\eta u \boldsymbol{x}$, which is Hebb learning rule. This learning rule does not include $d$, so it is an unsupervised learning rule without teacher guidance, and theory [18] verifies that it is unstable under certain conditions. The expanded type is

$$
\Delta w_{j}(k)=w_{j}(k)-w_{j}(k-1)=\eta_{j} u(k) x_{j}(k)
$$

where, in this paper, $u(k)=t_{\text {bon(norm) }}(k)$. Simulations show that this learning rule has strong "independence" and "self-learning ability", and its learning speed is very fast. So the output waveform has a fairly high response speed. However, because of the lack of teacher guidance, the steady-state errors are relatively large.

To better illustrate the 2 arguments above, a periodic square waveform is chosen as an example. The reasons for the choice are: 1) for periodic waveform, comparisons can be made between different waveforms or among different segments of the same waveform; 2) for square waveform, it has rising and falling edges and smooth segments, so the steepness of the former can be used to compare the response speed while the smoothness of the latter can be used to compare the steady-state errors. The simulated output waveform using perceptron learning rule is presented in Figure 5(a), which shows that the rising and falling edges are not steep (i.e., the response speed is slow) but the smooth segments are very flat (i.e., the steady-state errors are very small). The simulated output waveform using Hebb learning rule is presented in Figure 5(b), which shows that the rising and falling edges are steeper than those in Figure 5(a) (i.e., the response speed is faster), but there exist oscillations and great overshoots in the smooth segments (i.e., the steady-state errors are large); the oscillations seem to grow larger, implying the likelihood to become unstable.

Given that the strong point of perceptron learning rule is the weak point of Hebb learning rule and vice versa, the authors creatively combine them together and propose the perceptron-Hebb learning rule:

$$
\Delta w_{j}(k)=w_{j}(k)-w_{j}(k-1)=\eta_{j} e(k) u(k) x_{j}(k) .
$$

The simulated output waveform using the new learning rule is presented in 


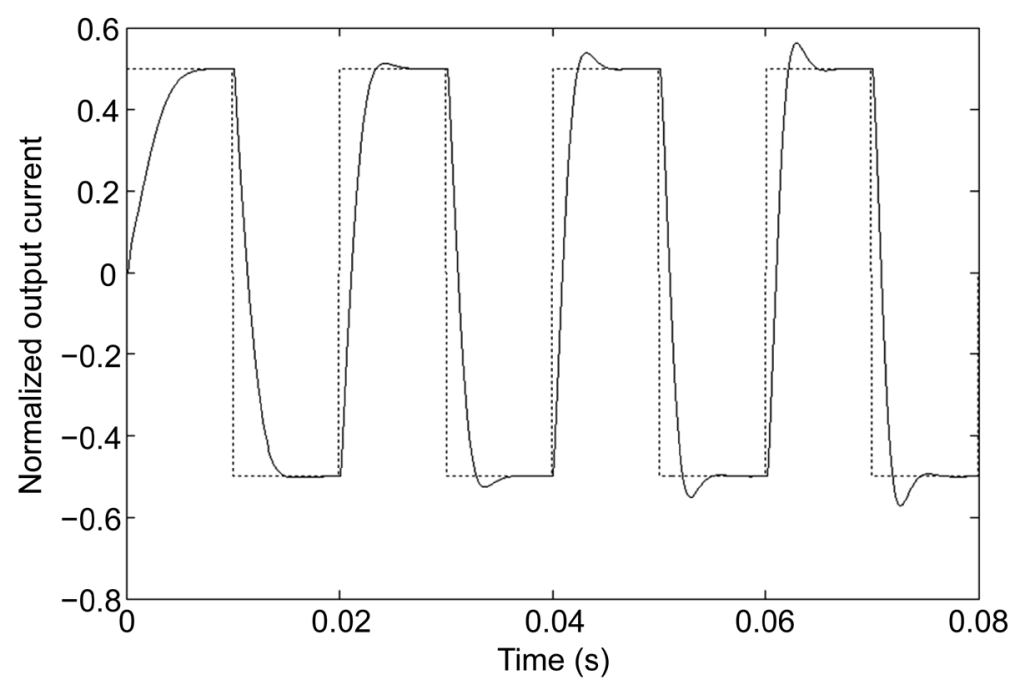

(a)

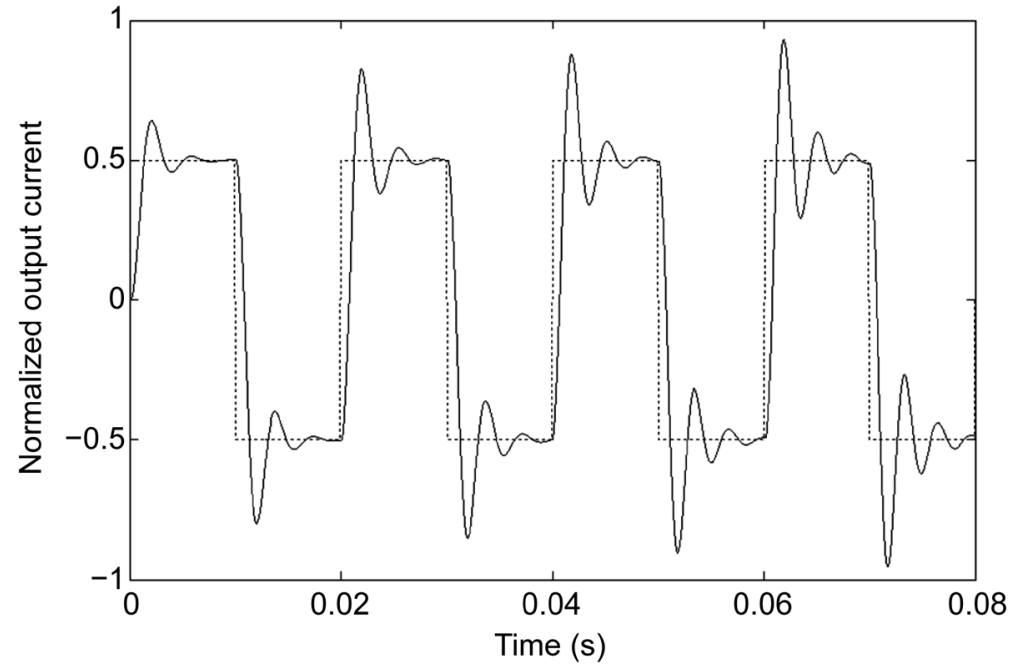

(b)

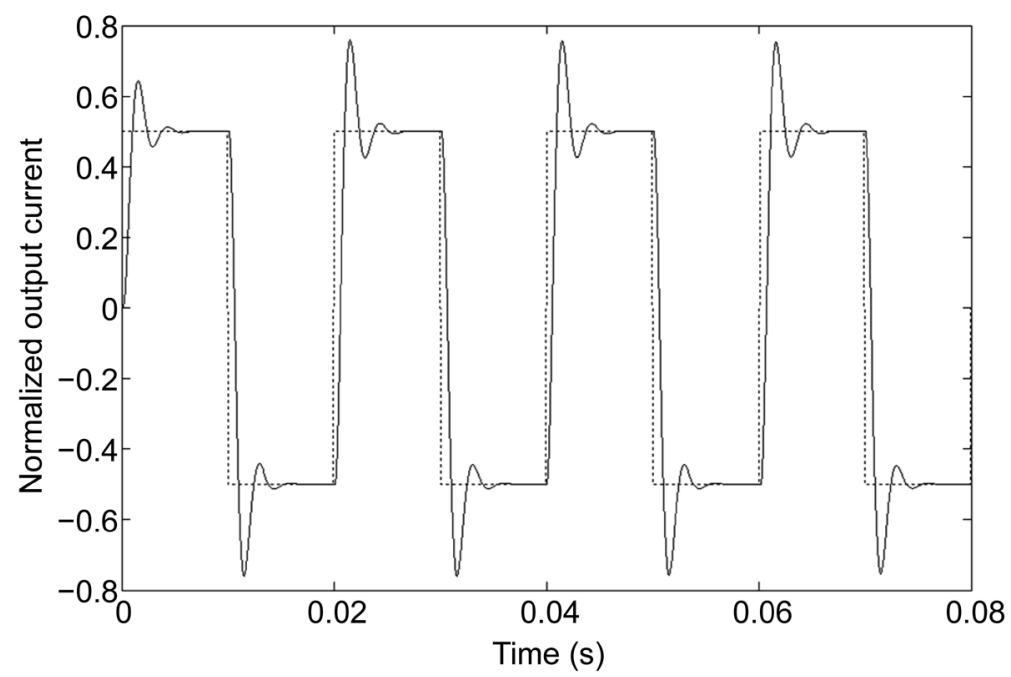

(c)

Figure 5. Comparison of the simulated output waveforms using 3 learning rules. (a) Perceptron learning rule, (b) Hebb learning rule, (c) perceptron Hebb learning rule. 
Figure 5(c), which shows that the rising and falling edges are steeper than those in Figure 5(a) and the smooth segments are flatter than those in Figure 5(b), meaning that both the response speed and the steady-state errors are improved-the new learning rule inherits the strong points of the two but gets rid of their weak points to a large extent; moreover, the possible unstability of Hebb learning rule never exists.

\subsection{Control Flow and Stability Analysis}

The control flow of single-neuron adaptive quasi-PID control method for simulation or actual control is summarized in Figure 6. It is shown that Equations (32), (41) and (36) are the 3 most important computational procedures of the flow chart, but they introduce only a small amount of floating additions and multiplications. These calculations are of relatively low computational complexities, meaning that the control method is very suitable for real-time control and for realization on low-end microprocessors.

From Equation (33), it is seen that $w_{j}(0)$ is actually initialized by quasi-PID parameters, which, as mentioned above, would vary during operation. So $w_{j}(0)$ is eventually initialized by values with small unpredictable errors. Therefore, it is necessary to analyze the impacts of the inaccuracy of the initial $w_{j}(0)$ on system stability. Considering the great complexity of the calculation process, only the conclusive results are presented.

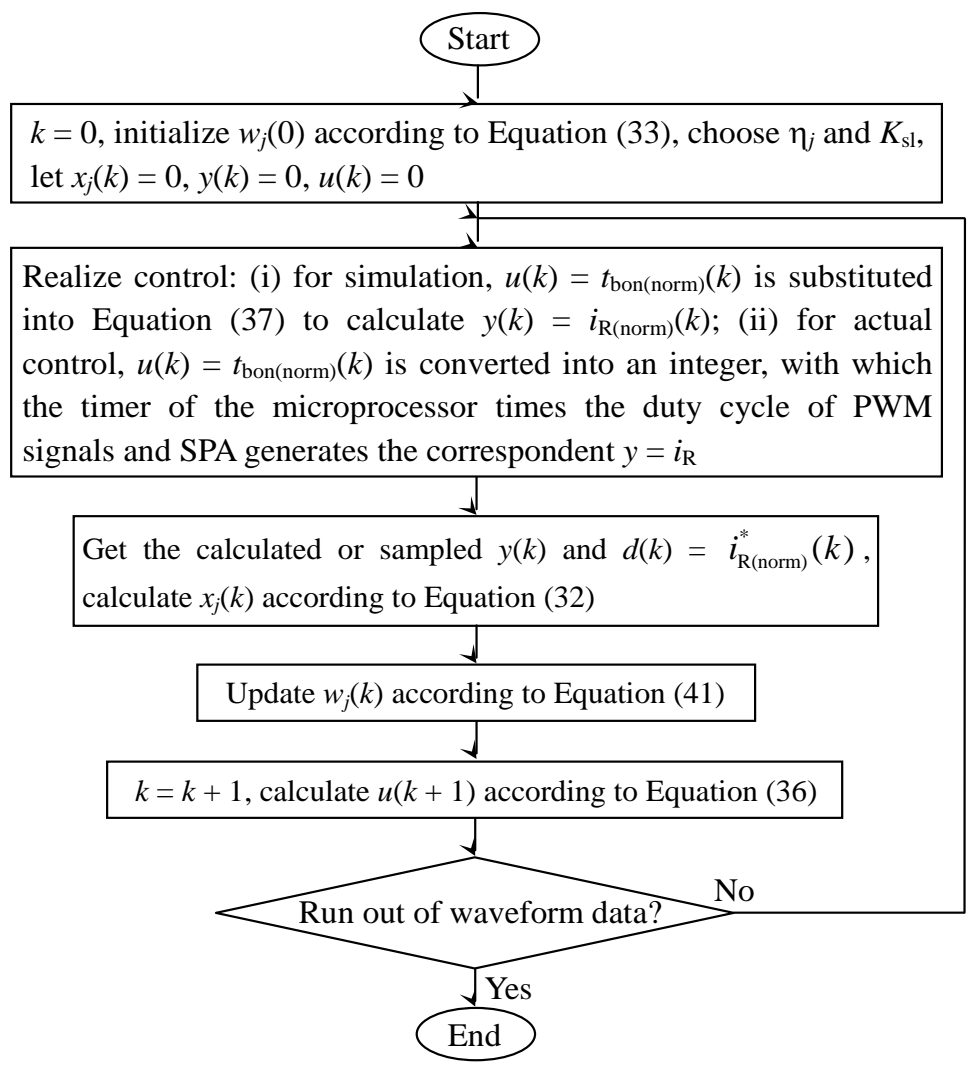

Figure 6. Flow chart of single-neuron adaptive quasi-PID control method for simulation or actual control. 
By performing $z$ transforms on Equations (36) and (37) respectively, then solving the resultant simultaneous equations, the system function $H(z)=I_{R(\text { norm })}(z) / I_{R(\text { norm })}^{*}(z)$ can be obtained. After rationalizations of both the numerator and the denominator polynomials of $H(z)$, the denominator polynomial becomes the characteristic polynomial that is in the following form:

$$
F_{c h}(z)=a_{5} z^{5}+a_{4} z^{4}+a_{3} z^{3}+a_{2} z^{2}+a_{1} z+a_{0}
$$

where $a_{0}, a_{1}, \cdots, a_{5}$ are the coefficients acquired from rationalizations. In terms of Jury criteria [19], the constraint conditions for system stability are as follows:

1) The first criterion requires $F_{c h}(1)>0$, and it is fulfilled straightforwardly because $a_{0}, a_{1}, \cdots, a_{5}$ are all positive numbers;

2) The order of $F_{c h}(z)$ is 5 , an odd number, so the second criterion requires $F_{c h}(-1)<0$, the calculation of which gives the in equation

$$
-0.00634 \bar{w}_{1}-0.1268 \bar{w}_{2}+0.2536 \bar{w}_{3}<2.3454 / K_{s l}
$$

3) The third criterion requires $\left|a_{0}\right|<a_{5}$, the calculation of which gives the in equation

$$
0.1845 K_{s l} \bar{w}_{3}<1
$$

4) ...

The punctuation "..." means the curtailment of the subsequent calculations. From calculations, it is found that as long as the choice of $K_{s l}$ fulfills both Equation (43) and Equation (44), the curtailed in equations are fulfilled as well; what's more, all the in equations have some margins to retain their inequalities, which not only gives the choice of $K_{s l}$ certain freedom, but also makes the impacts of the small unpredictable errors caused by variations of quasi-PID parameters on the initializations of $w_{j}(0)$ negligible. In short, the initializations of $w_{j}(0)$ according to Equation (33) and the choice of $K_{s l}$ according to in equations (43) and (44) can ensure the system stability.

\section{Simulation and Experimental Results}

In this section, the effectiveness of single-neuron adaptive quasi-PID control method is illustrated by 4 groups of simulation and experimental results. Section 5.1 tests the sheer ability of quasi-PID control method to counteract the inherent tracking error without the aid of the single neuron. The next 3 sections concentrate on testing the adaptabilities of single-neuron adaptive quasi-PID control method to different loads, operating conditions and disturbances.

\subsection{Ability to Counteract the Inherent Tracking Error}

A5A (RMS), $50 \mathrm{~Hz}$ sinusoidal waveform is chosen for the test. Here, in order to compare the actual performances of quasi-PID control method with the current command signal $i_{R}^{*}$ (with $i_{R}^{*}$, the control method is actually the PI control method [20]) and with the modified command current signal $\hat{i}_{R}^{*}$, the single neuron is temporarily thrown off. The results are presented in Figure 7, and it is clear that the simulated waveforms and the experimental ones are alike. Figure 7 


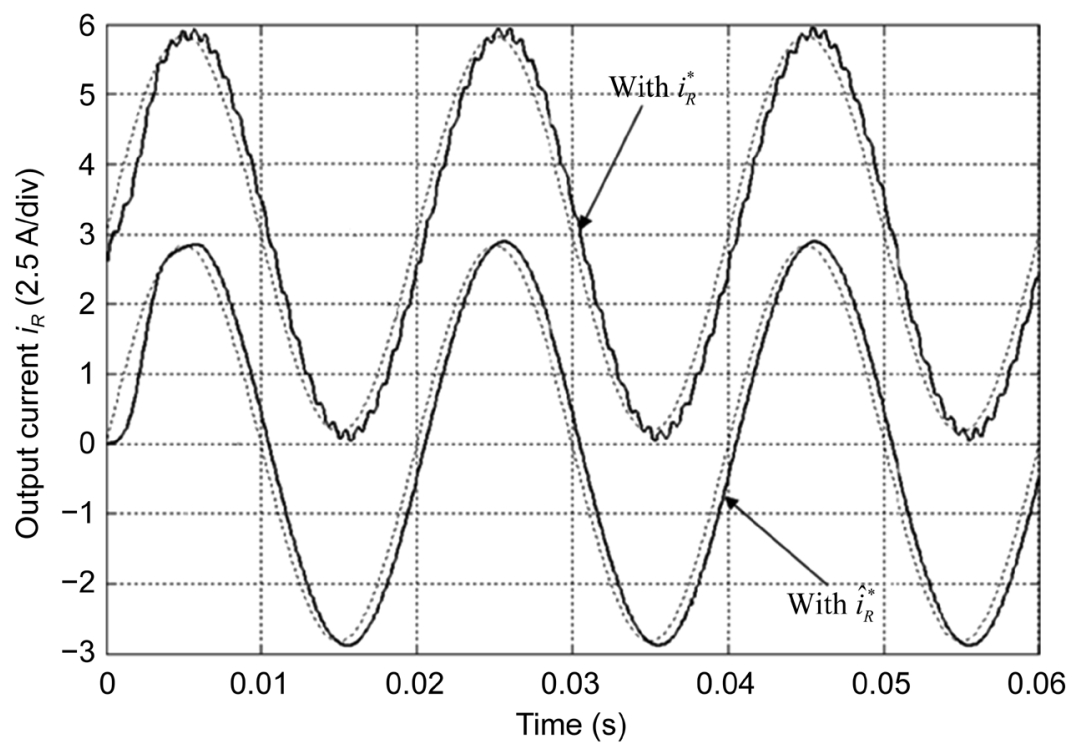

(a)

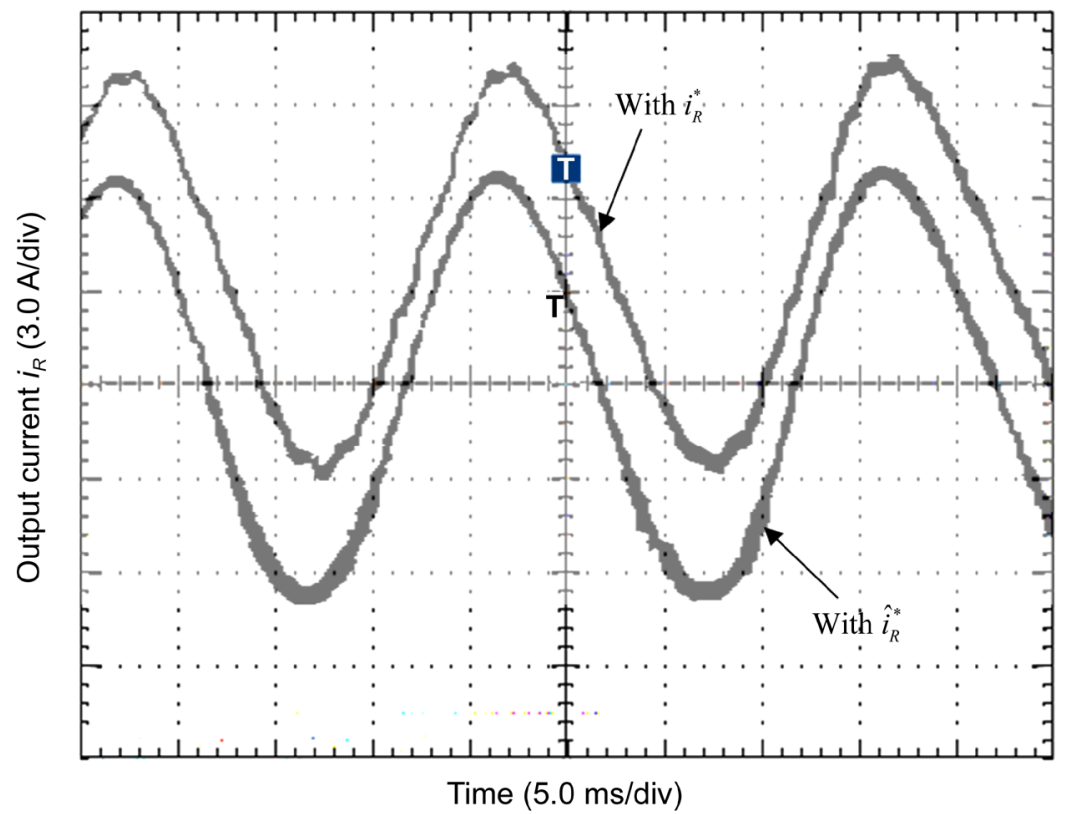

(b)

Figure 7. Ability of quasi-PID control method to counteract the inherent tracking error. (a) Simulation result, (b) experimental result.

shows that the output waveform with $i_{R}^{*}$ is fluctuating around the expected output waveform and is distorted in the vicinity of the peaks, which illustrates the existence and the detrimental effects of the inherent tracking error; however, the one with $\hat{i}_{R}^{*}$ satisfactorily inhibits the fluctuations and distortions, which means the inherent tracking error is effectively counteracted. At the end of this subsection, it should be pointed out that the experimental waveforms in Figure $7(\mathrm{~b})$ are obviously thicker than those in Figure 10(f), even the one with $\hat{i}_{R}^{*}$, which is the visible representation of the impacts of the circuit parameters drift and the random disturbances as mentioned in Section 4. These unpredictable 
errors can only be eliminated or compensated by the adaptability of the single neuron.

\subsection{Adaptability to Load Variations}

A $\pm 5 \mathrm{~A}$ (peak-to-peak value), $50 \mathrm{~Hz}$ square waveform (its characteristics were discussed in Subsection 4.2) is chosen to test the tracking speed (the response speed) and the tracking accuracy (the steady-state errors) of single-neuron adaptive quasi-PID control method. Normally, to current tracking, the load resistor $R$ is of a few ohms, so 2 situations, $R=3 \Omega$ and $10 \Omega$, are chosen for the test. The reason for the choice of this load difference $(10 \Omega-3 \Omega=7 \Omega)$ is that if the 2 values of $R$ are fairly close, the results would be too close to distinguish. However, this choice gives rise to a problem. The maximum output power of the prototype machine in this paper is designed as $100 \mathrm{~W}$. If the $\pm 5 \mathrm{~A}$ square waveform is outputted to $R=3 \Omega$, the maximum output power is at least $75 \mathrm{~W}$, and if outputted to $R=10 \Omega$, the maximum output power would be up to $250 \mathrm{~W}$, which is unrealizable for the prototype machine. Thus, only the simulation results are presented (shown in Figure 8).

A comparison of Figure 8(a) and Figure 8(b) shows that the steepness of the rising and falling edges and the smoothness of the smooth segments are alike except the overshoots, so the tracking speed and the tracking accuracy are almost invariant for different values of $R$, which illustrates the good adaptability of single-neuron adaptive quasi-PID control method to different load resistors.

\subsection{Adaptability to System Parameters Drift}

As mentioned in Section 4, there are many types of system parameters drift, so, for brevity, the drift of the load resistor $R$ at different temperatures is chosen as a test example, where it is assumed that $R$ varies from $3 \Omega$ to $5 \Omega$ with temperature increase. In practice, this variation is actually very slow, but for convenience the variation of $R$ is further assumed to be abrupt because the fast variation can encompass the slow variation as its special case. A5A (RMS), $50 \mathrm{~Hz}$ sinusoidal waveform is again chosen for the test instead of the square waveform, because as to a square waveform, choosing the abrupt variation point at the rising or falling edge would seem to be too special while choosing at the smooth segment would lack representativeness. It is unsafe to abruptly vary the load resistor by a switch or a relay on-line on the prototype machine, and the switch may introduce side effect to the circuit, so again, only the simulation results are presented (shown in Figure 9).

Figure 9(a) shows that $R$ varies abruptly from $3 \Omega$ to $5 \Omega$ at $0.042 \mathrm{~s}$, and the induced disturbance on the output waveform is nearly undetectable. For clear presentation, the dynamic tracking error $e_{i}$ is presented in Figure 9(b) to illustrate the disturbance. Figure 9(b) indirectly illustrates the rapid adjustment of single-neuron adaptive quasi-PID control method towards the abrupt disturbance. 


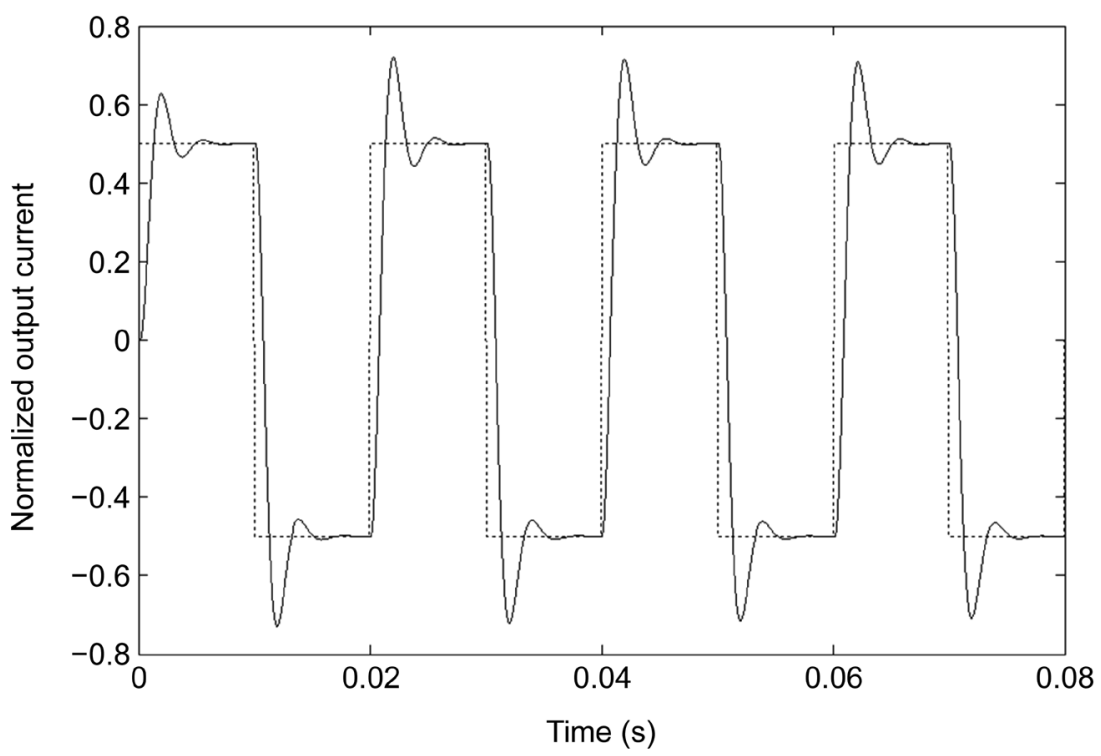

(a)

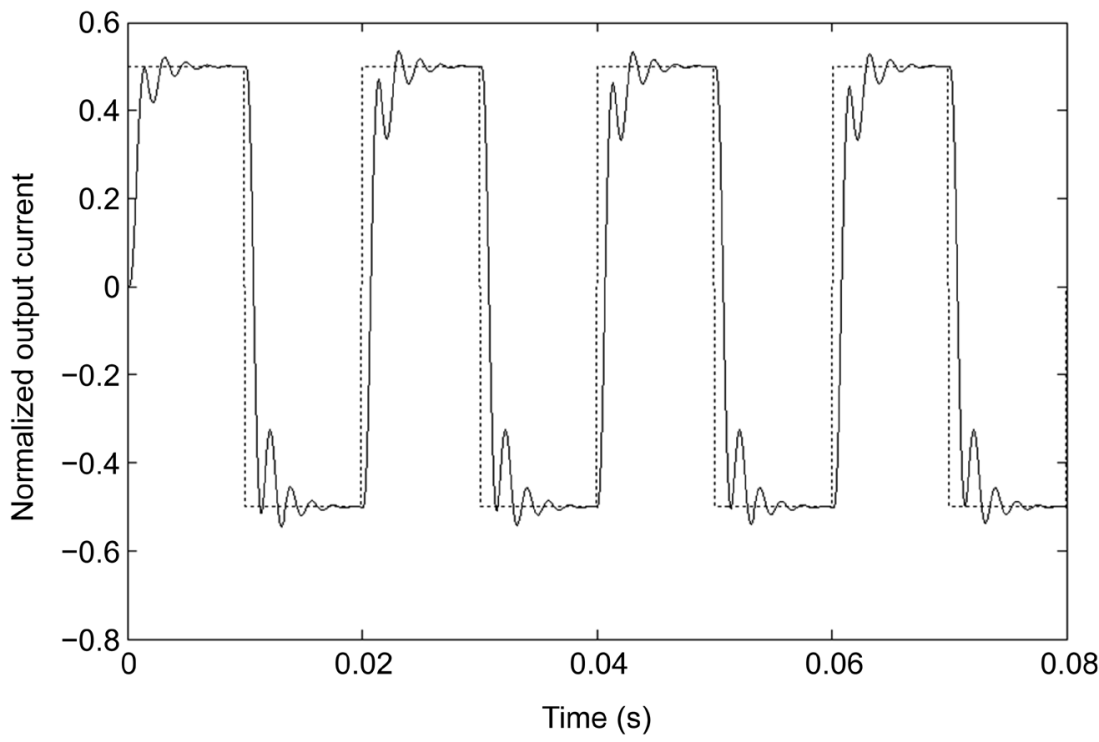

(b)

Figure 8. Adaptability of single-neuron adaptive quasi-PID control method to different load resistors. (a) $R=3 \Omega$, (b) $R=10 \Omega$.

\subsection{Adaptability to Waveforms with Different Frequency Components}

Different types of output waveforms contain different frequency components, the content and duration of which, in practice, may be unpredictable. Although the predesigned sampling frequency $f_{s}$ determined by the hardware fixes the theoretically maximum bandwidth of the open-loop system $\left(f_{s} / 2\right)$, the controller may lead to a great loss of the predesigned bandwidth of the close-loop system. From many simulations and experiments, the authors found that most of the control methods (such as those mentioned in Section 1) are more suitable for generating and amplifying sinusoidal waveforms or specified waveforms only, 


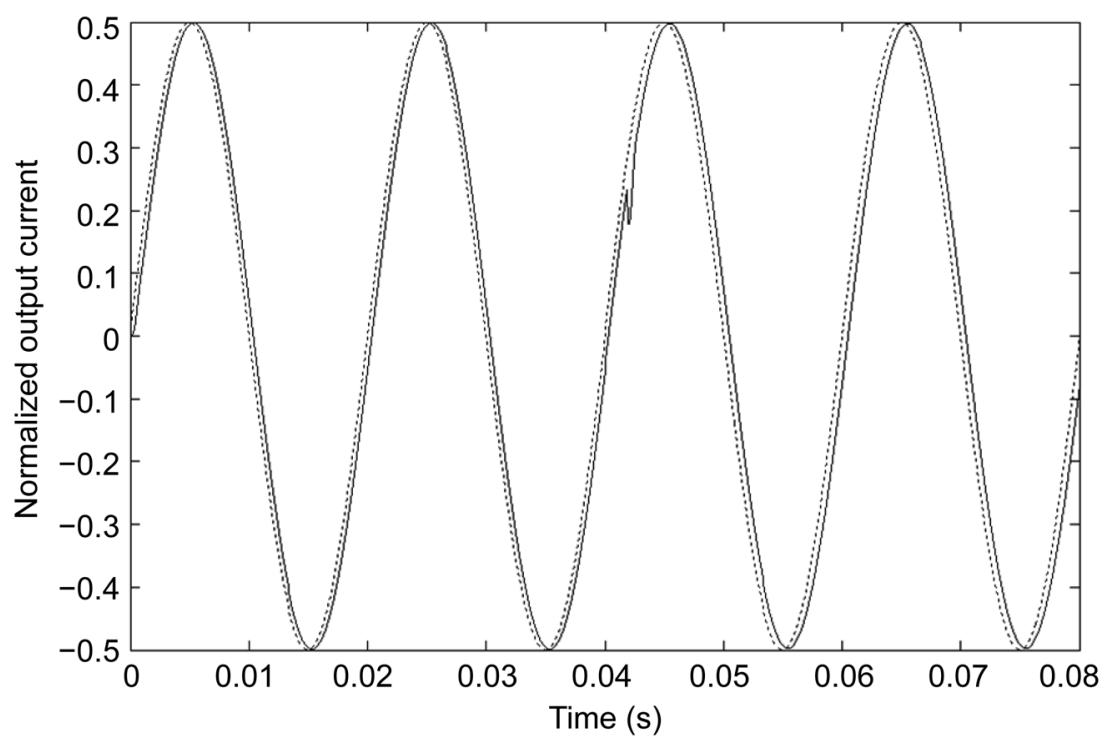

(a)

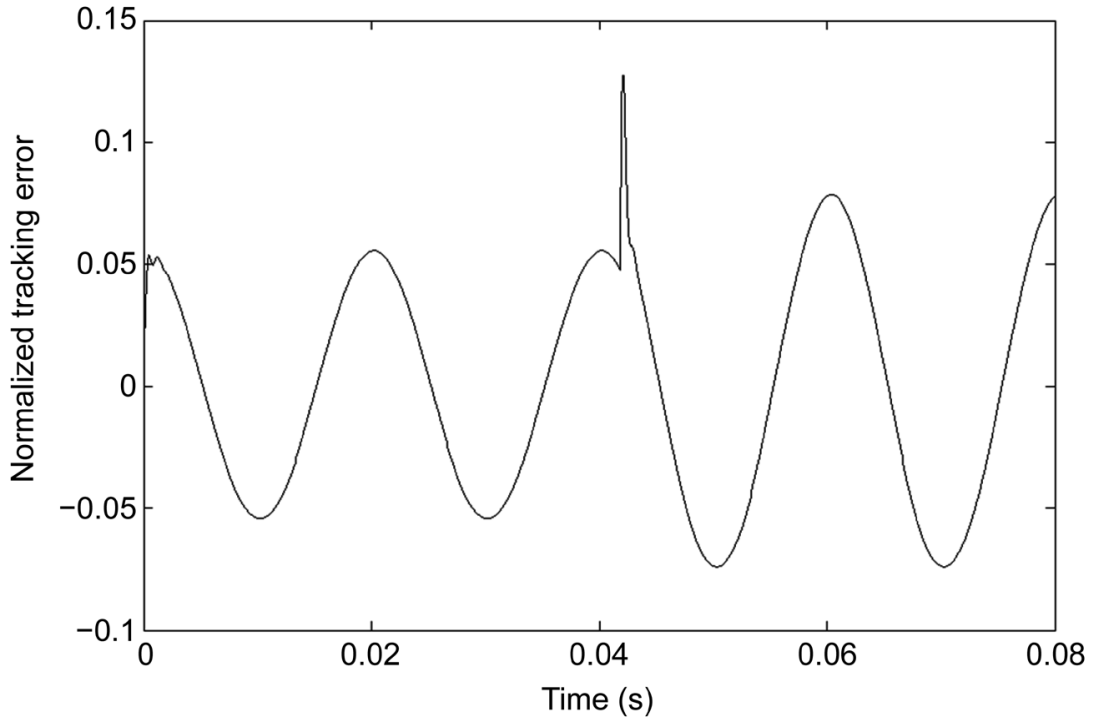

(b)

Figure 9. Adaptability of single-neuron adaptive quasi-PID control method to abrupt load variation. (a) The simulated actual output waveform, (b) dynamic tracking error.

since the frequency components of these waveforms are predetermined and the parameters of the controller can be directly adjusted towards these frequency components to acquire a relatively high and stable tracking accuracy; however, for waveforms with unpredictable frequency components, the tracking accuracy of these control methods may decline uncontrollably if there exist some frequency components not preconsidered during the design process of the controller due to the poor adaptability of these control methods. Thus, in this subsection, the adaptability of single-neuron adaptive quasi-PID control method to different types of waveforms, with and without unpredictable frequency components, is tested, and the simulation and experimental results are presented in Figure 10. 


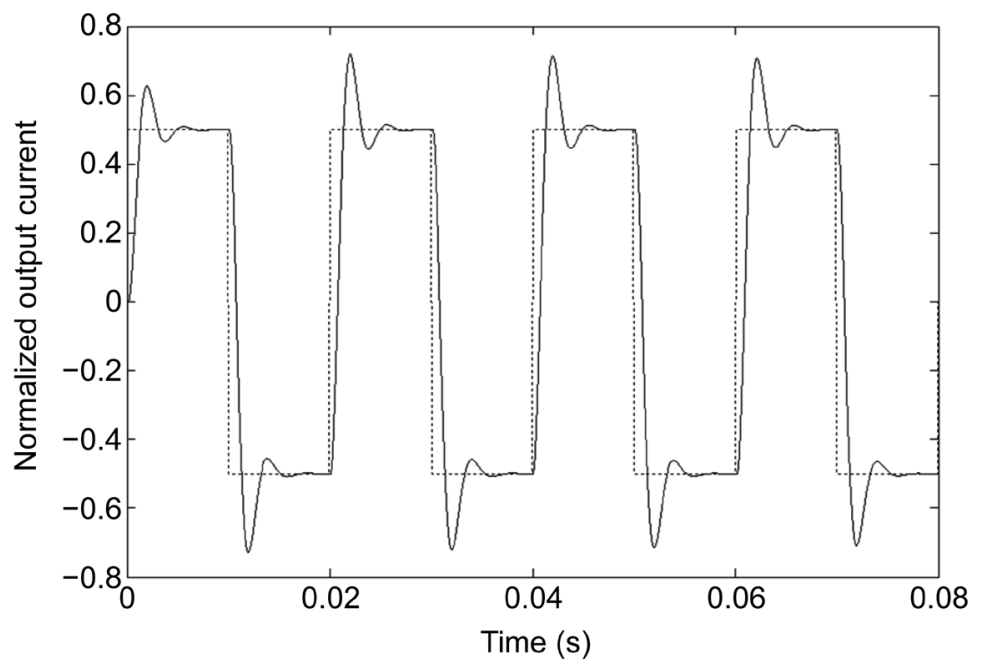

(a)

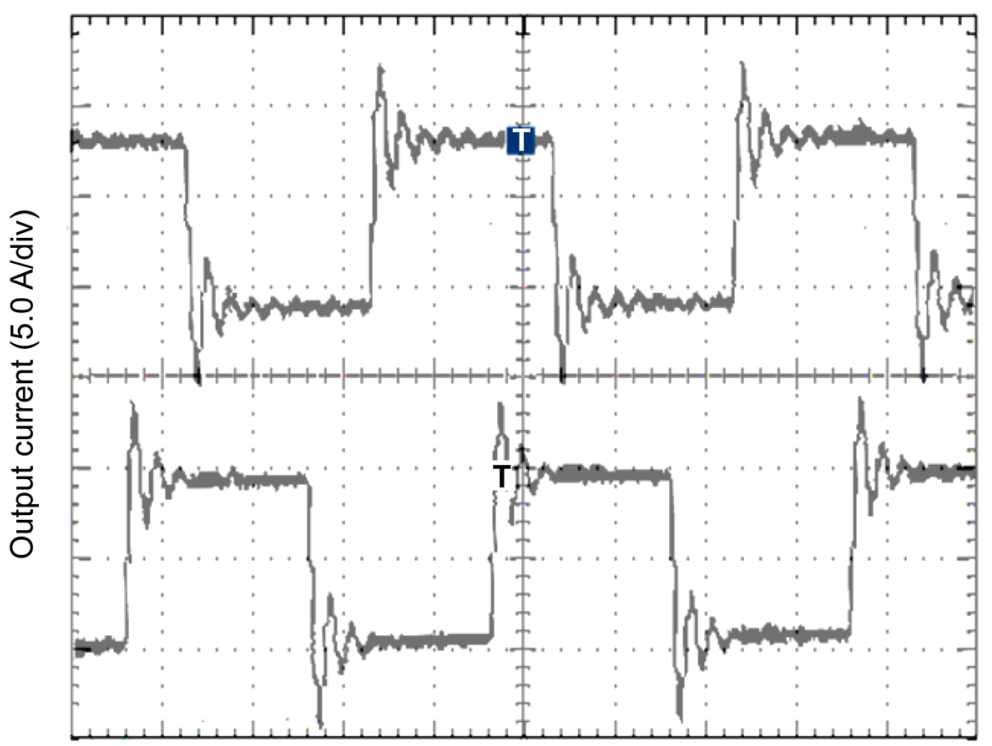

Time (5.0 ms/div)

(b)

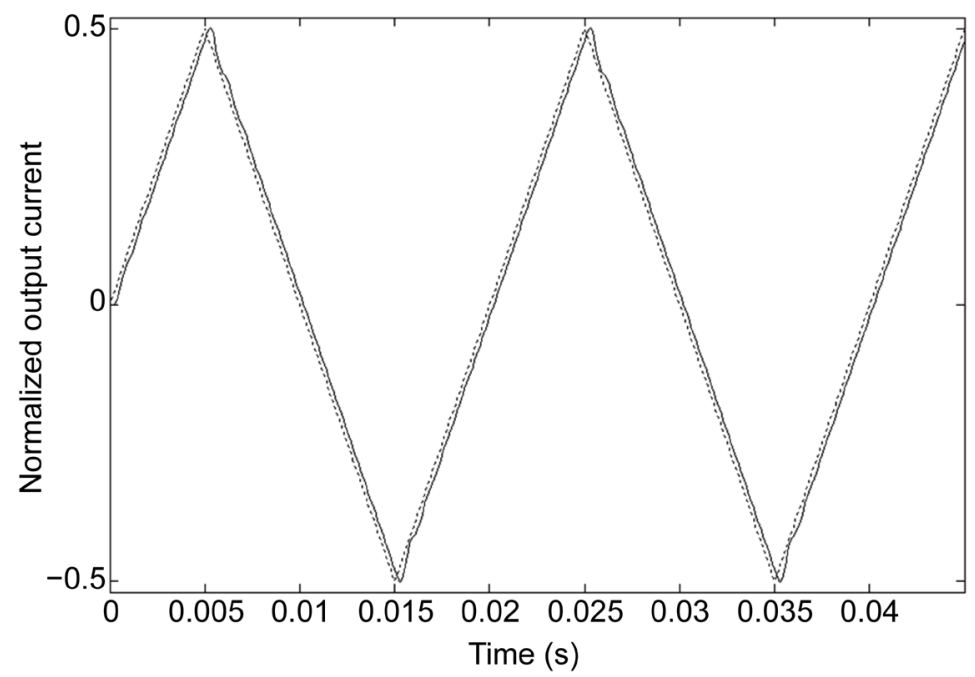

(c) 


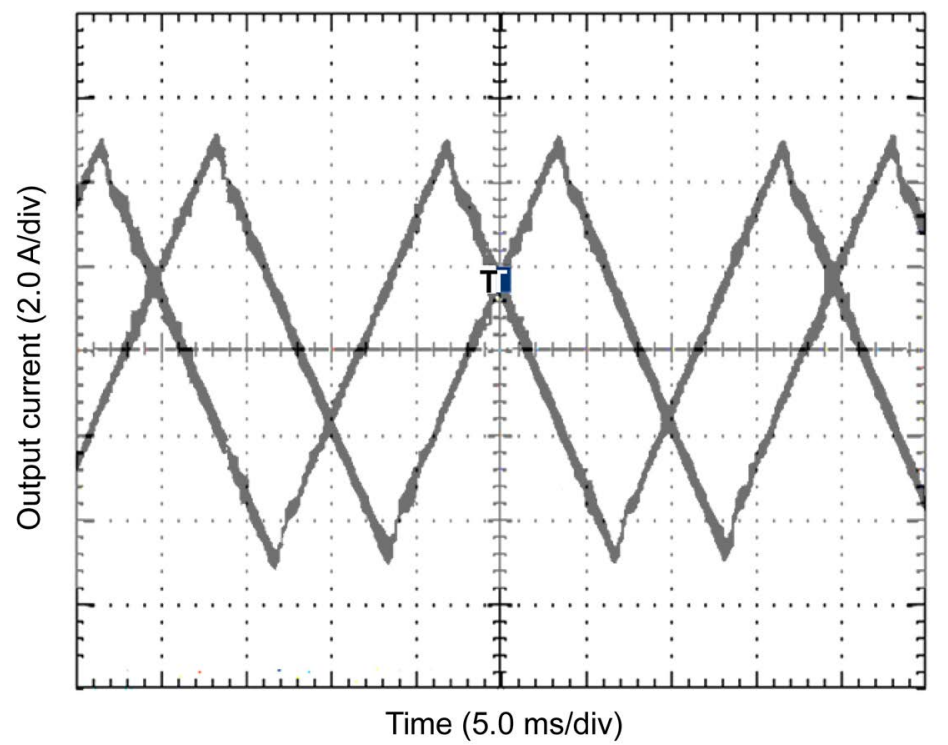

(d)

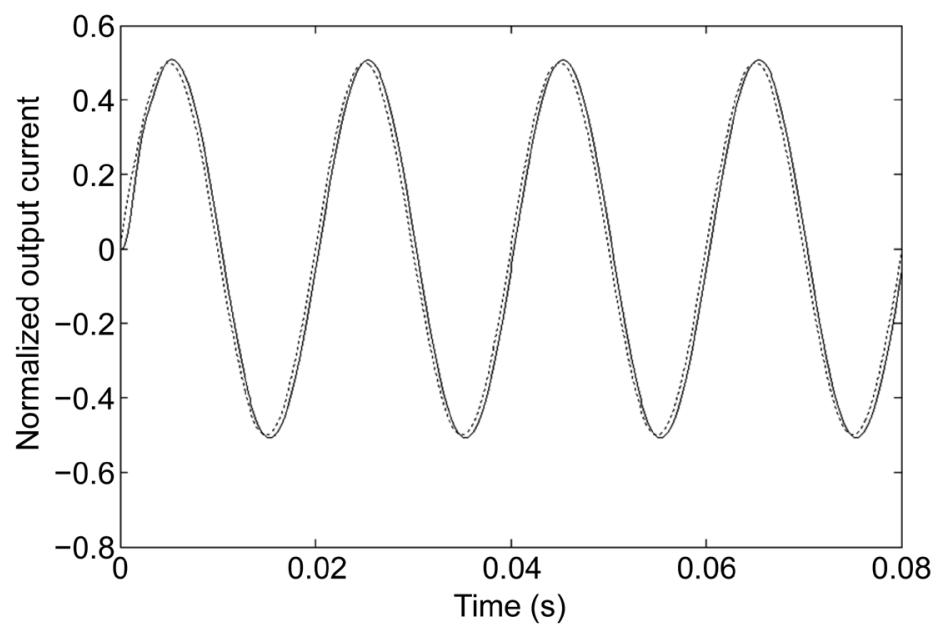

(e)

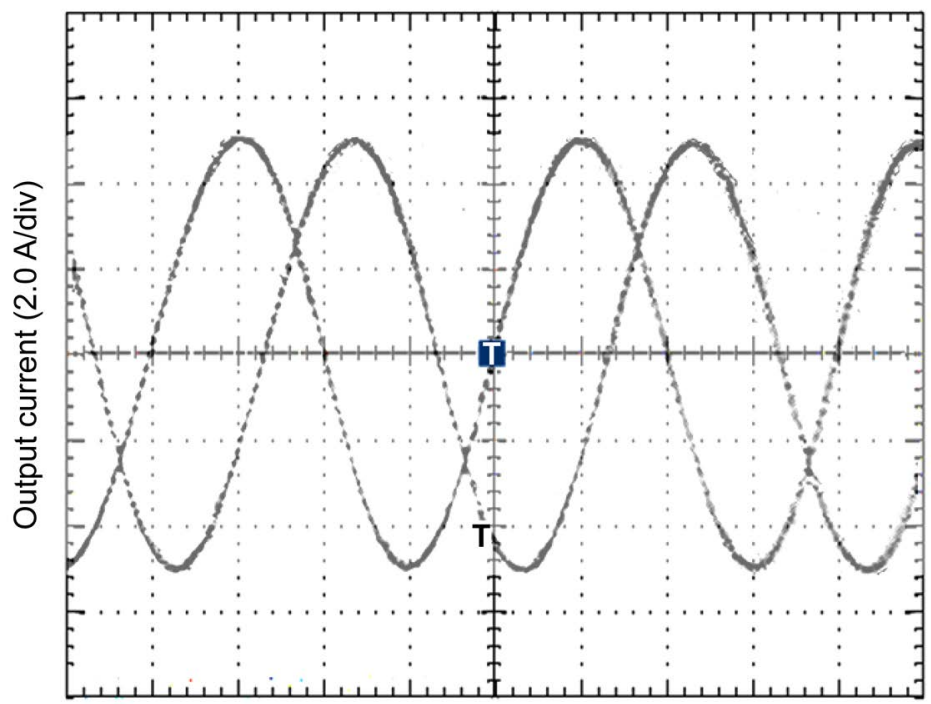

Time $(5.0 \mathrm{~ms} / \mathrm{div})$

(f) 


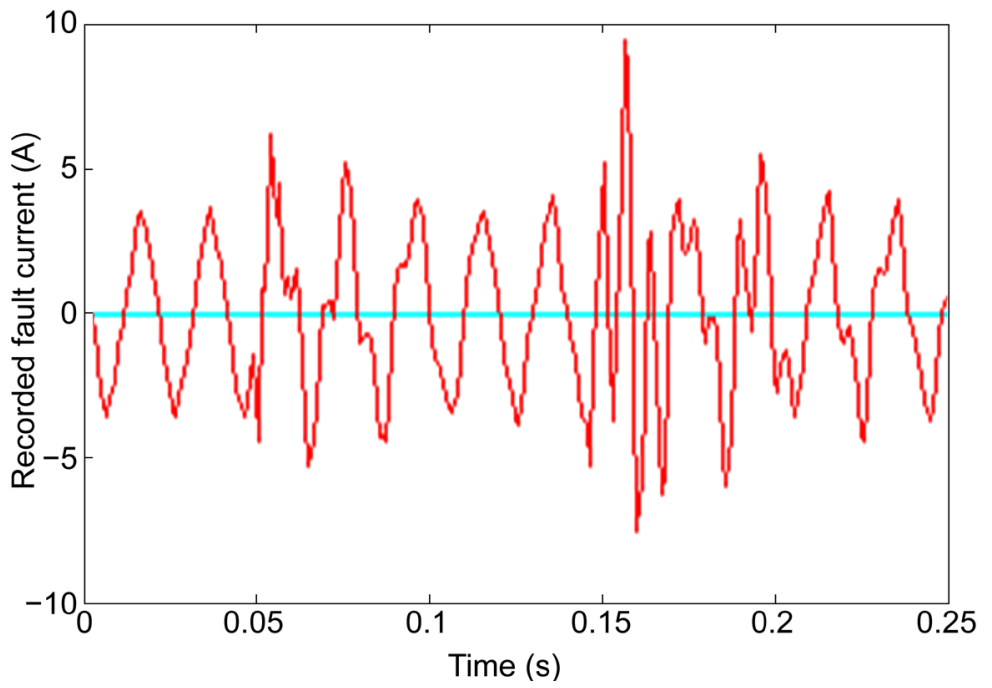

(g)

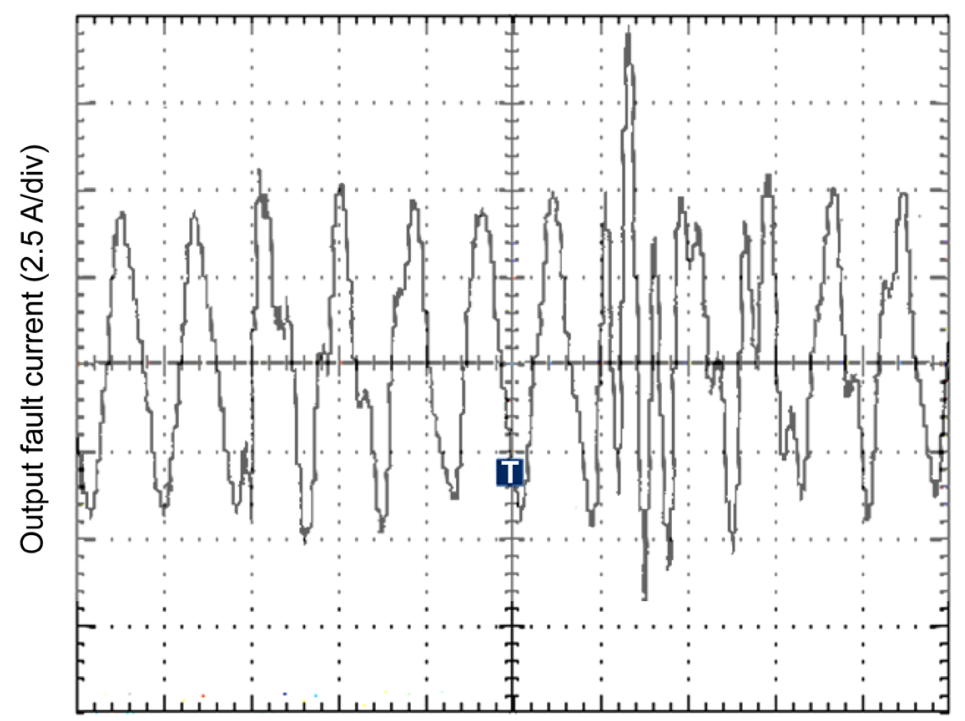

Time (25.0 ms/div)

(h)

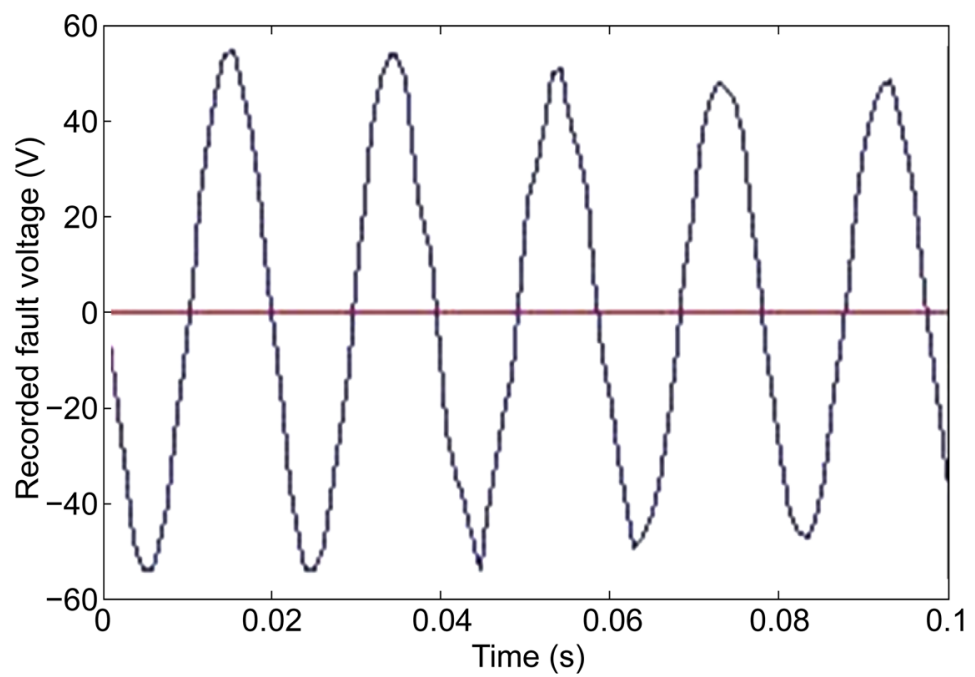

(i) 


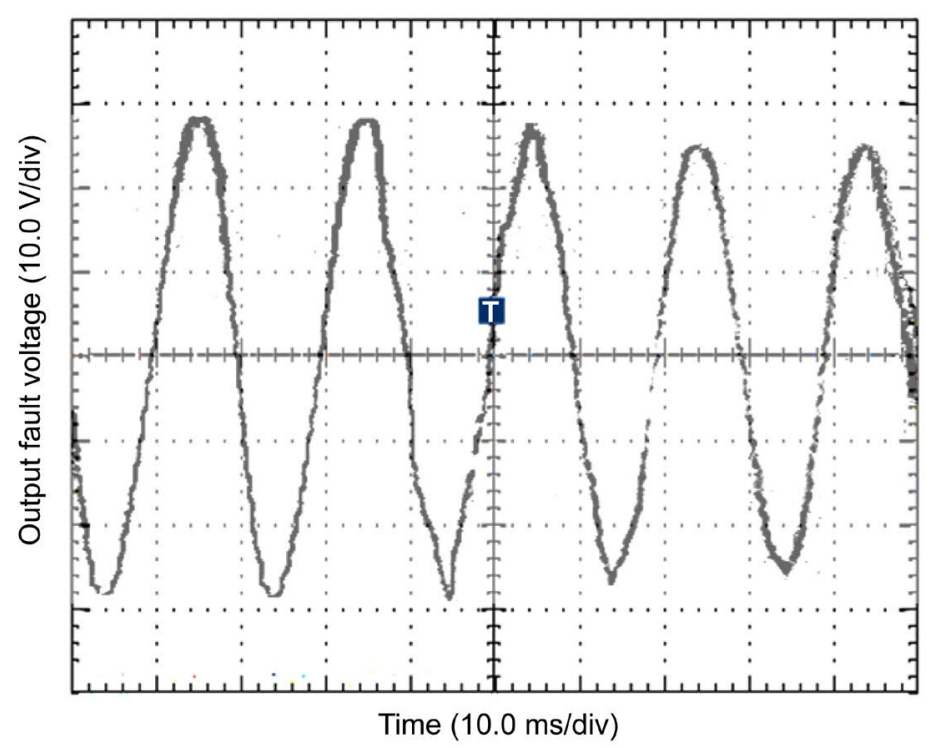

(j)

Figure 10. Adaptability of single-neuron adaptive quasi-PID control method to waveforms with and without unpredictable frequency components. (a) Simulated square waveform, (b) experimental square waveforms of phases $A$ and $B$, (c) simulated triangular waveform, (d) experimental triangular waveforms of phases $A$ and $B$, (e) simulated sinusoidal waveform, (f) experimental sinusoidal waveforms of phases $A$ and $B$, (g) a fault current waveform recorded by DFR, (h) experimental fault current waveform of (g), (i) a fault voltage waveform recorded by DFR, (j) experimental fault voltage waveform of (i).

Figures 10(a)-(f) show that the simulation waveforms and the experimental waveforms match with each other closely, and Figures $10(\mathrm{~g})-(\mathrm{j})$ show that the experimental fault current waveform and the experimental fault voltage waveform match the corresponding ones recorded by digital fault recorder (DFR) satisfactorily. The waveforms in Figures 10(a)-(f) contain invariable (deterministic) frequency components that can be obtained by Fourier series expansion, and the waveforms in Figures $10(\mathrm{~g})$-(j) contain variable (unpredictable) frequency components, the unpredictability of which is generated by the random characteristics of the faults in electrical power systems. These figures illustrate excellent adaptabilities of single-neuron adaptive quasi-PID control method to waveforms with deterministic and unpredictable frequency components from qualitative angle. A new comparison of Figure 10(f) and Figure 7(b), which is correspondent to the one in Subsection 5.4, shows that the sinusoidal waveform in Figure 10(f) is slimmer and smoother than that in Figure 7(b), demonstrating that single-neuron adaptive quasi-PID control method adaptively inhibits the circuit parameters drift and the random disturbances.

However, merely assessing the accuracy of the output waveforms from a qualitative angle, i.e., from the subjective impression, is very superficial, especially when the waveform is too complex to discriminate its subtle discrepancies. Therefore, a quantitative criterion for accuracy assessment is constructed, which is able to assess the accuracy of the waveforms by making point-to- 
point comparisons between the actual output waveform and the expected one and then give a score. This quantitative criterion is mean square error $(M S E) e_{M S E}=E\left[\left(i_{R}^{*}-i_{R}\right)^{2}\right]$, where the notation $E[\cdot]$ signifies the mathematical expectation computation. For discrete time series, $E[\cdot]$ is estimated by a statistical quantity:

$$
\hat{\varepsilon}_{M S E} \approx \frac{1}{N} \sum_{k=1}^{N}\left[i_{R(\text { norm })}^{*}(k)-i_{R(\text { norm })}(k)\right]^{2} \times 100 \%
$$

where $N$ is the length of the time series. As an example, Equation (45) is performed on the experimental fault current waveform in Figure 10(h), which is a waveform with unpredictable frequency components, and the result is $\hat{\varepsilon}_{\text {MSE }} \approx 0.11 \%$. For comparison, the same fault current waveform is generated by quasi-PID control method (without the single neuron), and the result is $\hat{\varepsilon}_{\text {MSE }} \approx 2.9 \%$; by PI control method, $\hat{\varepsilon}_{\text {MSE }} \approx 5.7 \%$; by repetitive control method, $\hat{\varepsilon}_{\text {MSE }} \approx 4.5 \%$; by deadbeat control method, $\hat{\varepsilon}_{\text {MSE }} \approx 2.4 \%$; by sliding mode control method, $\hat{\varepsilon}_{\text {MSE }} \approx 2.2 \%$. It is clear that with the introduction of the quasi- $D$ term, quasi-PID control method gains a relatively high accuracy by compensating the inherent tracing error of PI control method, which, to some extent, is already comparable to the accuracies of deadbeat control method and sliding mode control method. And the improvement owing to the single neuron is more impressive, and this high accuracy has made the applications of SPAs in some of the high-accuracy fields possible. For example, the regenerated fault current and fault voltage in Figure 10(h) and Figure 10(j) can be used for protective relay test or other similar tests [21], the accuracy requirement of which is generally prescribed as $\leq 1.5 \%$; this means that by virtue of single-neuron adaptive quasiPID control method SPAs can also be used in protective relay test equipment so as to make the equipment small in volume and weight but high in performance.

\section{Conclusions}

1) Quasi-PID control method that is directly derived from the circuit topology of SPA can effectively inhibit the inherent tracking error, and its derivation process reveals an important fact: the quasi- $D$ term is not a real $D$ term in PID control method, so PID control method is actually not suitable for the control; because the real $D$ term may serve as a weird disturbance causing system unstability, that is why few references reported such an application. 2) Although quasi-PID control method may suffer from quasi-PID parameters variations caused by circuit parameters drift and random disturbances, it can be combined with a single neuron to form single-neuron adaptive quasi-PID control method to maintain its excellence. 3) Simulation and experimental results illustrate that single-neuron adaptive quasi-PID control method is able to accurately track both the predictable and the unpredictable waveforms, and the quantitative analysis demonstrates that its accuracy is higher than most of the modern control methods and is comparable to that of LPAs. 4) Compared with many modern control methods, the programming realization of single-neuron adaptive quasi-PID control method is very simple, and the computational complexity is very small. 


\section{Acknowledgements}

This research is supported by Chongqing Education Committee Science and Technology Research Project (KJ1603605) and Yongchuan District Science and Technology Committee Natural Science Fund Project (Ycstc, 2016nc3001).

\section{References}

[1] Gong, G.H., Ertl, H. and Kolar, J.W. (2008) Novel Tracking Power Supply for Linear Power Amplifiers. IEEE Transactions on Industrial Electronics, 55, 684-698. https://doi.org/10.1109/TIE.2007.896152

[2] Hornik, T. and Zhong, Q.C. (2011) A Current-Control Strategy for Voltage-Source Inverters in Microgrids Based on $\mathrm{H}$ and Repetitive Control. IEEE Transactions on Power Electronics, 26, 943-952. https://doi.org/10.1109/TPEL.2010.2089471

[3] Zhang, B., Wang, D.W., Zhou, K.L. and Wang, Y.G. (2008) Linear Phase Lead Compensation Repetitive Control of a CVCF PWM Inverter. IEEE Transactions on Industrial Electronics, 55, 1595-1602. https://doi.org/10.1109/TIE.2008.917105

[4] Hassan, T.K. (2011) A Repetitive-PI Current Controller for Boost Single Phase PFC Converters. Energy and Power Engineering, 3, 69-78.

https://doi.org/10.4236/epe.2011.32010

[5] Nishida, K., Rukonuzzman, M. and Nakaoka, M. (2004) Advanced Current Control Implementation with Robust Deadbeat Algorithm for Shunt Single-Phase Voltage-Source Type Active Power Filter. IEEE Proceedings of Electric Power Applications, 151, 283-288. https://doi.org/10.1049/ip-epa:20040317

[6] Mohamed, Y.A.R.I. and El-Saadany, E.F. (2007) An Improved Deadbeat Current Control Scheme with a Novel Adaptive Self-Tuning Load Model for a Three-Phase PWM Voltage-Source Inverter. IEEE Transactions on Industrial Electronics, 54, 747-759. https://doi.org/10.1109/TIE.2007.891767

[7] Guldemir, H. (2011) Study of Sliding Mode Control of Dc-Dc Buck Converter. Energy and Power Engineering, 3, 401-406. https://doi.org/10.4236/epe.2011.34051

[8] Benamor, A., Chrifialaui, L., Messaound, H. and Chaabane, M. (2011) Sliding Mode Control, with Integrator, for a Class MIMO Nonlinear Systems. Energy and Power Engineering, 3, 435-444. https://doi.org/10.4236/eng.2011.35050

[9] Guo, L.P., John, Y.H. and Nelms, R.M. (2011) Comparative Evaluation of Sliding Mode Fuzzy Controller and PID Controller for a Boost Converter. Electric Power Systems Research, 81, 99-106. https://doi.org/10.1016/j.epsr.2010.07.018

[10] Sun, X.M., Long, H.L., Liu, D.C. and Li, P. (2009) Multi-Objective Optimization Design for Snubber in Switching Power Amplifier. International Conference on Electrical Machines and Systems, Tokyo, 15-18 November 2009, 1-6. https://doi.org/10.1109/icems.2009.5382697

[11] Itoh, Y., Hori, N. and Kamei, H. (2004) Digital Redesign of a Nonlinear State-Feedback Control System Based on the Principle of Equivalent Areas. Proceedings of the SICE Annual Conference, 1, 350-354.

[12] Sakamoto, T. and Hori, N. (2002) New PWM Schemes Based on the Principle of Equivalent Areas. Proceedings of the IEEE International Symposium on Industrial Electronics, 2, 505-510. https://doi.org/10.1109/isie.2002.1026341

[13] Farahani, H.F. and Sarabadani, H. (2011) Modulation Index Effect on the 5-Level SHE-PWM Voltage Source Inverter. Energy and Power Engineering, 3, 187-194. https://doi.org/10.4236/eng.2011.32022

[14] Routimo, M., Salo, M. and Tuusa, H. (2007) Comparison of Voltage-Source and 
Current-Source Shunt Active Power Filters. IEEE Transactions on Power Electronics, 22, 636-643. https://doi.org/10.1109/TPEL.2006.890005

[15] Franklin, G.F., Powell, J.D. and Naeini, A.E. (2007) Feedback Control of Dynamic Systems.5th Edition, Posts and Telecom Press, Beijing.

[16] Kress, R. (1998) Numerical Analysis. Springer, New York. https://doi.org/10.1007/978-1-4612-0599-9

[17] Amari, S.I. (1990) Mathematical Foundations of Neurocomputing. Proceedings of the IEEE, 78, 1443-1463. https://doi.org/10.1109/5.58324

[18] Haykin, S. (2002) Adaptive Filter Theory. 4th Edition, Prentice Hall, Upper Saddle River.

[19] Jonckheere, E. and Ma, C. (1989) A Further Simplification to Jury's Stability Test. IEEE Transactions Circuits and Systems, 36, 463-464. https://doi.org/10.1109/31.17599

[20] Dixon, J., Tepper, S. and Moran, L. (1996) Practical Evaluation of Different Modulation Techniques for Current-Controlled Voltage Source Inverters. IEE Proceedings of Electric Power Applications, 143, 301-306. https://doi.org/10.1049/ip-epa:19960411

[21] Sun, X.M., Long, H.L. and Liu, D.C. (2008) The Design of an Electrical Fault Waveform Regenerator. International Conference on Electrical Machines and Systems, Wuhan, 17-20 October 2008, 136-141.

\section{Appendix A. Circuit Components List}

Digital Signal Processor (DSP): TMS320 LF2407A

IGBT Module: PM30CSJ060

Fast-Recovery Free-Wheeling Diode: HFA04TB60

DC Energy-Storage Capacitor: $4700 \mu \mathrm{F}$

$\bar{V}_{d c}: 67 \mathrm{~V}$

$L: 1.8 \mathrm{mH}$

C: $37.6 \mu \mathrm{F}$

R: $0-4 \Omega$

$T_{s}: 1 \times 10^{-4} \mathrm{~s}$

$f_{s}: 10 \mathrm{kHz}$ 
Submit or recommend next manuscript to SCIRP and we will provide best service for you:

Accepting pre-submission inquiries through Email, Facebook, LinkedIn, Twitter, etc. A wide selection of journals (inclusive of 9 subjects, more than 200 journals)

Providing 24-hour high-quality service

User-friendly online submission system

Fair and swift peer-review system

Efficient typesetting and proofreading procedure

Display of the result of downloads and visits, as well as the number of cited articles Maximum dissemination of your research work

Submit your manuscript at: http://papersubmission.scirp.org/

Or contact jpee@scirp.org 